\title{
Energy-dependent noncommutative quantum mechanics
}

\author{
Tiberiu Harko ${ }^{1,2,3, a}$, Shi-Dong Liang ${ }^{1,4, b}$ \\ ${ }^{1}$ School of Physics, Sun Yat-Sen University, Guangzhou 510275, People's Republic of China \\ 2 Department of Physics, Babes-Bolyai University, Kogalniceanu Street, 400084 Cluj-Napoca, Romania \\ ${ }^{3}$ Department of Mathematics, University College London, Gower Street, London WC1E 6BT, UK \\ ${ }^{4}$ State Key Laboratory of Optoelectronic Material and Technology, and Guangdong Province Key Laboratory of Display Material and Technology, \\ Sun Yat-Sen University, Guangzhou 510275, People's Republic of China
}

Received: 15 January 2019 / Accepted: 15 March 2019 / Published online: 3 April 2019

(C) The Author(s) 2019

\begin{abstract}
We propose a model of dynamical noncommutative quantum mechanics in which the noncommutative strengths, describing the properties of the commutation relations of the coordinate and momenta, respectively, are arbitrary energy-dependent functions. The Schrödinger equation in the energy-dependent noncommutative algebra is derived for a two-dimensional system for an arbitrary potential. The resulting equation reduces in the small energy limit to the standard quantum mechanical one, while for large energies the effects of the noncommutativity become important. We investigate in detail three cases, in which the noncommutative strengths are determined by an independent energy scale, related to the vacuum quantum fluctuations, by the particle energy, and by a quantum operator representation, respectively. Specifically, in our study we assume an arbitrary power-law energy dependence of the noncommutative strength parameters, and of their algebra. In this case, in the quantum operator representation, the Schrö dinger equation can be formulated mathematically as a fractional differential equation. For all our three models we analyze the quantum evolution of the free particle, and of the harmonic oscillator, respectively. The general solutions of the noncommutative Schrödinger equation as well as the expressions of the energy levels are explicitly obtained.
\end{abstract}

\section{Contents}

1 Introduction . . . . . . . . . . . . . 1

2 Energy-dependent noncommutative geometry and algebra . . . 5

3 The Schrödinger equation in the energy-dependent noncommutative geometry . . . . . . . . . .

\footnotetext{
a e-mail: t.harko@ucl.ac.uk

be-mail: stslsd@mail.sysu.edu.cn
}

3.1 Probability current and density in the energydependent potential . . . . . . . . . 7

3.2 The free particle . . . . . . . . . . 8

3.3 The harmonic oscillator . . . . . . . . . . 8

4 Physical mechanisms generating energy-dependent noncommutative algebras, and their implications . . 9

4.1 The noncommutative algebra of the spacetime quantum fluctuations (SQF) model . . . . . . . 9

4.2 The noncommutative algebra of the energy coupling (EC) model . . . . . . . . . . . 10

4.3 The noncommutative algebra of the energy operator $(\mathrm{EO})$ model . . . . . . . . . . . . 10

5 Quantum evolution in the spacetime quantum fluctuation (SQF) energy-dependent noncommutative model . . . . . 11

5.1 Quantum mechanics of the free particle in the SQF model .............. . . 11

5.2 The harmonic oscillator . . . . . . . . . 12

6 Quantum dynamics in the energy coupling model . . 12

6.1 Quantum evolution: the Schrödinger equation . 12

6.2 Quantum evolution: wave function and energy levels . . . . 13

6.3 The case of the free particle . . . . . . . . . . 14

6.4 The harmonic oscillator . . . . . . . . . 15

7 Quantum evolution in the energy operator (EO) energy-dependent noncommutative geometry . . . . 16

7.1 Fractional calculus: a brief review . . . . . . 16

7.2 The fractional Schrödinger equation . . . . . 17

7.3 The free particle: the case $\alpha=1$. . . . . 18

8 Discussions and final remarks ......... . 18

References............... 21

\section{Introduction}

It is generally believed today that the description of the spacetime as a manifold $M$, locally modeled as a flat Minkowski space $M_{0}=\mathbb{R} \times \mathbb{R}^{3}$, may break down at very short dis- 
tances of the order of the Planck length $l_{P}=\sqrt{G \hbar / c^{3}} \approx$ $1.6 \times 10^{-33} \mathrm{~cm}$, where $G, \hbar$ and $c$ are the gravitational constant, Planck's constant, and the speed of light, respectively [1]. This assumption is substantiated by a number of arguments, following from quantum mechanical and general relativistic considerations, which point towards the impossibility of an arbitrarily precise location of a physical particle in terms of points in spacetime.

One of the basic principles of quantum mechanics, Heisenberg's uncertainty principle, requires that a localization $\Delta x$ in spacetime can be reached by a momentum transfer of the order of $\Delta p=\hbar / \Delta x$, and an energy of the order of $\Delta E=$ $\hbar c / \Delta x$ [2-4]. On the other hand, the energy $\Delta E$ must contain a mass $\Delta m$, which, according to Einstein's general theory of relativity, generates a gravitational field. If this gravitational field is so strong that it can completely screen out from observations some regions of spacetime, then its size must be of the order of its Schwarzschild radius $\Delta R \approx G \Delta m / c^{2}$. Hence we easily find $\Delta R \approx G \Delta E / c^{4}=G \hbar / c^{3} \Delta x$, giving $\Delta R \Delta x \approx G \hbar / c^{3}$. Thus the Planck length appears to give the lower quantum mechanically limit of the accuracy of position measurements [5]. Therefore the combination of the Heisenberg uncertainty principle with Einstein's theory of general relativity leads to the conclusion that at short distances the standard concept of space and time may lose any operational meaning.

On the other hand, the very existence of the Planck length requires that the mathematical concepts for high-energy (short distance) physics have to be modified. This follows from the fact that classical geometrical notions and concepts may not be well suited for the description of physical phenomena at very short distances. Moreover, some drastic changes are expected in the physics near the Planck scale, with one important and intriguing effect being the emergence of the noncommutative structure of the spacetime. The basic idea behind spacetime noncommutativity is very much inspired by quantum mechanics. A quantum phase space is defined by replacing canonical position and momentum variables $x^{\mu}, p^{\nu}$ with Hermitian operators that obey the Heisenberg commutation relations,

$\left[\widehat{x}^{\mu}, \widehat{p}^{\nu}\right]=i \hbar \delta^{\mu \nu}$

Hence the phase space becomes smeared out, and the notion of a point is replaced with that of a Planck cell. The generalization of commutation relations for the canonical operators (coordinate-momentum or creation-annihilation operators) to non-trivial commutation relations for the coordinate operators was performed in [6,7], where it was first suggested that the coordinates $x^{\mu}$ may be noncommutating operators, with the six commutators being given by $\left[\widehat{x}_{\mu}, \widehat{x}_{\nu}\right]=i \frac{a^{2}}{\hbar} L_{\mu \nu}$,

where $a$ is a basic length unit, and $L_{\mu \nu}$ are the generators of the Lorentz group. In this approach, Lorentz covariance is maintained, but the translational invariance is lost. A rigorous mathematical approach to noncommutative geometry was introduced in [8-11], by generalizing the notion of a differential structure to arbitrary $C^{*}$ algebras, as well as to quantum groups and matrix pseudo-groups. This approach led to an operator algebraic description of noncommutative spacetimes, based entirely on algebras of functions.

Since at the quantum level noncommutative spacetimes do appear naturally when gravitational effects are taken into account, their existence must also follow from string theory. In [12] it was shown that if open strings have allowed endpoints on D-branes in a constant $B$-field background, then the endpoints live on a noncommutative space with the commutation relations

$\left[\widehat{x}^{\mu}, \widehat{x}^{\nu}\right]=i \theta^{\mu \nu}$,

where $\theta^{\mu \nu}$ is an antisymmetric constant matrix, with components $c$-numbers with the dimensionality (length) ${ }^{-2}$. More generally, a similar relation can also be imposed on the particle momenta, which generates a noncommutative algebra in the momentum space of the form

$\left[\widehat{p}_{\mu}, \widehat{p}_{\nu}\right]=i \eta_{\mu \nu}$,

where $\eta_{\mu \nu}$ are constants. In [13] noncommutative field theories with commutator of the coordinates of the form $\left[x^{\mu}, x^{\nu}\right]=i \Lambda^{\mu \nu}{ }_{\omega} x^{\omega}$ have been studied. By considering $\Lambda \mathrm{a}$ Lorentz tensor, explicit Lorentz invariance is maintained, a free quantum field theory is not affected. On the other hand, since invariance under translations is broken, the conservation of energy-momentum tensor is violated, and a new law expressed by a Poincaré-invariant equation is obtained. The $\lambda \phi^{4}$ quantum field theory was also considered. It turns out that the usual UV divergent terms are still present in this model. Moreover, new type of terms also emerge that are IR divergent, violates momentum conservation and lead to corrections to the dispersion relations.

The physical implications and the mathematical properties of the noncommutative geometry have been extensively investigated in [14-42]. In the case when $\left[\widehat{p}_{i}, \widehat{p}_{j}\right]=0$, the noncommutative quantum mechanics goes into the usual one, described by the non-relativistic Schrödinger equation,

$H(\tilde{x}, p) \psi(\tilde{x})=E \psi(\tilde{x})$, 
where $\tilde{x}^{\mu}=x^{\mu}-(1 / 2) \theta^{\mu v} p_{v}$ [43]. In the presence of a constant magnetic field $B$ and an arbitrary central potential $V(r)$, with Hamiltonian

$\widehat{H}=\frac{\widehat{p}^{2}}{2 m}+V(r)$,

the operators $\widehat{p}, \widehat{x}$ obey the commutation relations [43]

$\left[\widehat{x}^{1}, \widehat{x}^{2}\right]=i \theta, \quad\left[\widehat{x}^{\mu}, \widehat{p}_{\nu}\right]=i \hbar \delta_{\nu}^{\mu}, \quad\left[\widehat{p}_{1}, \widehat{p}_{2}\right]=i \frac{e}{c} B$.

Several other types of noncommutativity, extending the canonical one, have also been proposed. For example, in [44], a three-dimensional noncommutative quantum mechanical system with mixing spatial and spin degrees of freedom was investigated. In this study it was assumed that the noncommutative spatial coordinates $\widehat{x}^{i}$, the conjugate momenta $\widehat{p}^{i}$, and the spin variables $\hat{s}^{i}$ obey the nonstandard Heisenberg algebra

$\left[\widehat{x}^{i}, \widehat{x}^{j}\right]=i \theta^{2} \epsilon^{i j k} \widehat{s}^{k}, \quad\left[\widehat{x}^{i}, \widehat{p}_{j}\right]=i \delta_{j}^{i}, \quad\left[\widehat{p}_{i}, \widehat{p}_{j}\right]=0$,

and

$\left[\widehat{x}^{i}, \widehat{s}^{j}\right]=i \theta \epsilon^{i j k \widehat{s}^{k}}, \quad\left[\widehat{s}^{i}, \widehat{s}^{j}\right]=i \epsilon^{i j k \widehat{s}^{k}}$,

respectively, where $\theta \in \mathbb{R}$ is the parameter of the noncommutativity. A classical model of spin noncommutativity was investigated in [45]. In the nonrelativistic case, the Poisson brackets between the coordinates are proportional to the spin angular momentum. The quantization of the model leads to the noncommutativity with mixed spatial and spin degrees of freedom. A modified Pauli equation, describing a spin half particle in an external electromagnetic field was also obtained, and it was shown that in spite of the presence of noncommutativity and nonlocality, the model is Lorentz invariant. Other physical and mathematical implications of spin noncommutativity were investigated in [46-48]

A model of dynamic position-dependent noncommutativity, involving the complete algebra of noncommutative coordinates

$\left[\widehat{x}^{\mu}, \widehat{x}^{\nu}\right]=i \omega^{\mu \nu}(\widehat{x})$

was proposed in [49], and further investigated in [50]. In [50] a system consisting of two interrelated parts was analyzed. The first describes the physical degrees of freedom with the coordinates $x^{1}$ and $x^{2}$, while the second corresponds to the noncommutativity $\eta$, which has a proper dynamics. It turns out that after quantization, the commutator of two physical coordinates is proportional to an arbitrary function of $\eta$. An interesting feature of this model is the dependence of nonlocality on the energy of the system, so that the increase of the energy leads to the increase in nonlocality. The physical properties of systems with dynamic noncommutativity were considered in [51-58].

A quantum mechanical system on a noncommutative space for which the structure constant is explicitly timedependent was investigated in [59], in a two-dimensional space with nonvanishing commutators for the coordinates $X, Y$ and momenta $P_{x}, P_{y}$ given by

$$
\begin{aligned}
{[X, Y] } & =i \theta(t),\left[P_{x}, P_{y}\right]=i \Omega(t), \\
{\left[X, P_{x}\right] } & =\left[Y, P_{y}\right]=i \hbar+i \frac{\theta(t) \Omega(t)}{4 \hbar} .
\end{aligned}
$$

Any autonomous Hamiltonian on such a space acquires a time-dependent form in terms of the conventional canonical variables. A generalized version of Heisenberg's uncertainty relations for which the lower bound becomes a time-dependent function of the background fields was also obtained. For a two-dimensional harmonic oscillator, after performing the Bopp shift, the Hamiltonian becomes times dependent, and $t$ is given by [59]

$$
\begin{aligned}
H(t)= & \frac{1}{2 m_{e}(t)}\left(p_{x}^{2}+p_{y}^{2}\right) \\
& +\frac{k_{e}(t)}{2}\left(x^{2}+y^{2}\right)+B_{e}(t) L_{z},
\end{aligned}
$$

where

$$
\begin{aligned}
\frac{1}{m_{e}(t)} & =\frac{1}{m}+\frac{m \omega^{2}}{4 \hbar^{2}} \theta^{2}(t), k_{e}(t)=m \omega^{2}+\frac{\Omega^{2}(t)}{4 m \hbar^{2}}, \\
B_{e} & =\frac{m \omega^{2} \theta(t)}{2 \hbar}+\frac{\Omega(t)}{2 \hbar m}, L_{z}=\left(p_{x} y-x p_{y}\right) .
\end{aligned}
$$

From a general physical point of view we can interpret the noncommutativity parameters $\theta^{\mu \nu}$ and $\eta_{\mu \nu}$ as describing the strength of the noncommutative effects exerted in an interaction. In this sense they are the analogues of the coupling constants in standard quantum field theory.

It is a fundamental assumption in quantum field theory that the properties of a physical system (including the underlying force laws) change when viewed at different distance scales, and these changes are energy dependent. This is the fundamental idea of the renormalization group method, which has found fundamental applications in quantum field theory, elementary particle physics, condensed matter etc. [60].

It is the main goal of the present paper to introduce and analyze a dynamic noncommutative model of quantum mechanics, in which the noncommutative strengths $\theta^{\mu \nu}$ and $\eta_{\mu \nu}$ are energy-dependent quantities. This would imply the existence of several noncommutative scales that range from the energy level of the standard model, where the lowenergy scales of the physical systems reduce the general noncommutative algebra to the standard Heisenberg algebra, and ordinary quantum mechanics, to the Planck energy scale. On energy scales of the order of the Planck energy, $E_{P}=\sqrt{\hbar c^{5} / G} \approx 1.22 \times 10^{19} \mathrm{GeV}$, the noncommutative 
effects become maximal. Under the assumption of the energy dependence of the noncommutative parameters, with the help of the generalized Seiberg-Witten map, we obtain the general form of the Schrödinger equation describing the quantum evolution in an energy-dependent geometry. The noncommutative effects can be included in the equation via a generalized quantum potential, which contains an effective (analogue) magnetic field, and an effective elastic constant, whose functional forms are determined by the energy-dependent noncommutative strengths.

The possibility of an energy-dependent Schrödinger equation was first suggested by Pauli [61], and it was further considered and investigated extensively (see [62-67] and the references therein). Generally, the nonlinearity induced by the energy dependence requires modifications of the standard rules of quantum mechanics [62]. In the case of a linear energy dependence of the potential for confining potentials the saturation of the spectrum is observed, which implies that with the increase of the quantum numbers the eigenvalues reach an upper limit [65]. The energy-dependent Schrödinger equation was applied to the description of heavy quark systems in [63], where for a linear energy dependence the harmonic oscillator was studied as an example of a system admitting analytical solutions. A new quark interaction was derived in [64], by means of a Tamm-Dancoff reduction, from an effective field theory constituent quark model. The obtained interaction is nonlocal and energy dependent. Moreover, it becomes positive and rises up to a maximum value when the interquark distance increases. The quantum mechanical formalism for systems featuring energydependent potentials was extended to systems described by generalized Schrödinger equations that include a positiondependent mass in [67]. Modifications of the probability density and of the probability current need the adjustments in the scalar product and the norm. The obtained results have been applied to the energy-dependent modifications of the Mathews-Lakshmanan oscillator, and to the generalized Swanson system.

From a physical point of view we can assume that the energy dependent noncommutative effects can be described by two distinct energy scales. One is the energy scale of the spacetime quantum fluctuations, generated by the vacuum background and the zero point energy of the quantum fields. The noncommutativity is then essentially determined by this energy scale, which is independent of the particle energy. This is the first explicit model we are considering, a two energy scales model, in which the energy of the quantum fluctuations and the particle energy evolve in different and independent ways. The alternative possibility, in which the noncommutative strengths are dependent on the particle energy only, is also investigated. We consider the quantum evolution of the free particle and of the harmonic oscillator in these cases, and the resulting energy spectrum and wave functions are determined. The particle oscillation frequencies are either dependent on the vacuum fluctuation energy scale, or they have an explicit dependence on the particle energy. In the limiting case of small energies we recover the standard results of quantum mechanics.

As a simple application of the developed general formalism we consider the case in which the noncommutative strengths $\eta$ and $\theta$ are power-law functions of energy, with arbitrary exponents. However, the quantization of such systems, in which we associate an operator to the energy, requires the mathematical/physical interpretation of operators of the form $\partial^{\alpha} / \partial t^{\alpha}$, where $\alpha$ can have arbitrary real) values, like, for example, $\alpha=1 / 2, \alpha=5 / 4$ etc. These types of problems belong to the field of fractional calculus [71-73], whose physical applications have been intensively investigated. In particular, the mathematical and physical properties of the fractional Schrö dinger equation, whose introduction was based on a purely phenomenological or abstract mathematical approach, have been considered in detail in [74-93]. It is interesting to note that the present approach gives a physical foundation for the mathematical use of fractional derivatives in quantum mechanics, as resulting from the noncommutative and energy-dependent structure of the spacetime. We present in full detail the fractional Schrödinger equations obtained by using two distinct quantization of the energy (the time operator and the Hamiltonian operator approach, respectively), and we investigate the quantum evolution of the free particle and of the harmonic oscillator in the time operator formalism for a particular simple choice of the energy dependent noncommutativity strength parameters.

The present paper is organized as follows. We introduce the energy-dependent noncommutative quantum geometry, its corresponding algebra, and the Seiberg-Witten map that allows one to construct the noncommutative set of variables from the commutative ones in Sect. 2. The Schrö dinger equation describing the quantum evolution in the energydependent noncommutative geometry is obtained in Sect. 3, where the form of the effective potential induced by the noncommutative effects is also obtained. Three relevant physical and mathematical mechanisms that could induce energydependent quantum behaviors in noncommutative geometry are discussed in Sect. 4, and their properties are explored in the framework of a particular model in which the noncommutativity parameters have a power-law dependence on energy. The quantum dynamics of a free particle and of the harmonic oscillator in the spacetime quantum fluctuations model is analyzed in Sect. 5, while the same physical systems are analyzed in the energy coupling model in Sect. 6 . The fractional Schrödinger equations for the quantum evolution of general quantum systems in the energy operator approach are presented in Sect. 7, where the dynamics of the free particles and of the harmonic oscillator are analyzed in 
detail. A brief review of the fractional calculus is also presented. We discuss and conclude our results in Sect. 8 .

\section{Energy-dependent noncommutative geometry and algebra}

In the present section we will introduce the basic definitions, conventions and relations for an energy-dependent generalization of the noncommutative geometry and algebra of physical variables, valid in the high-energy/small distance regime. In high-energy physics theoretical models where both the coordinate and momentum space noncommutativity is taken into account, in a four-dimensional space the coordinates and momenta satisfy the following algebra:

$\left[\widehat{x}^{\mu}, \widehat{x}^{\nu}\right]=i \theta^{\mu \nu}$,

$\left[\widehat{p}^{\mu}, \widehat{p}^{\nu}\right]=i \eta^{\mu \nu}$,

$\left[\widehat{x}^{\mu}, \widehat{p}^{\nu}\right]=i \hbar \Delta^{\mu \nu}$,

where the noncommutativity strength parameters $\theta^{\mu \nu}$ and $\eta^{\mu \nu}$ are antisymmetric. Due to the commutation relation given by Eq. (14c), this algebra is consistent with ordinary Quantum Mechanics. We shall assume in the following that the two matrices $\theta^{\mu \nu}$ and $\eta^{\mu \nu}$ are invertible, and moreover the matrix $\Sigma^{\mu \nu}=\delta^{\mu \nu}+\left(1 / \hbar^{2}\right) \theta^{\mu \alpha} \eta_{\alpha}^{\nu}$ is also invertible [26]. Under a linear transformation of the form

$\widehat{x}^{\mu}=A_{v}^{\mu} x^{\nu}+B_{v}^{\mu} p^{\nu}, \widehat{p}^{\mu}=C_{v}^{\mu} x^{\nu}+D_{v}^{\mu} p^{\nu}$,

also called the $D$ map, where A, B, C, and D are real constant matrices, the noncommutative algebra (14a)-(14c) can be mapped to the usual Heisenberg algebra, $\left[x^{\mu}, x^{\nu}\right]=0$, $\left[p^{\mu}, p^{\nu}\right]=0$, and $\left[x^{\mu}, p^{\nu}\right]=i \hbar \delta^{\mu \nu}$, respectively [26]. The matrices $\mathrm{A}, \mathrm{B}, \mathrm{C}$, and $\mathrm{D}$ satisfy the equations $\mathrm{AD}^{\mathrm{T}}-\mathrm{BC}^{\mathrm{T}}=$ $\mathrm{I}_{d \times d}, \mathrm{AB}^{\mathrm{T}}-\mathrm{BA}^{\mathrm{T}}=\Theta / \hbar$, and $\mathrm{CD}^{\mathrm{T}}-\mathrm{DC}^{\mathrm{T}}=\mathrm{N} / \hbar[26]$, where $\Theta$ and $N$ are matrices with the entries $\theta^{\mu \nu}$ and $\eta^{\mu \nu}$, respectively [26]. Due to the linear transformations (14d), the noncommutative algebra (14a)-(14c) admits a Hilbert space representation of ordinary quantum mechanics. However, it is important to note that the $D$ map is not unique.

In the present paper we generalize the algebra given by Eqs. (14a)-(14c) to the case when the parameters $\theta^{\mu v}$ and $\eta^{\mu \nu}$ are energy-dependent functions, so that

$\theta^{\mu \nu}=\theta^{\mu \nu}(E)$

and

$\eta^{\mu \nu}=\eta^{\mu \nu}(E)$,

respectively, where $E$ is a general energy parameter whose physical interpretation depends on the concrete physical problem under consideration.
In the following we will not consider time-like noncommutative relations, that is, we take $\theta^{0 i}=0$ and $\eta^{0 i}=0$, since otherwise the corresponding quantum field theory is not unitary.

The parameters $\theta$ and $\eta$ from Eqs. (14a)-(14c) can be represented generally as

$$
\begin{aligned}
\left(\theta^{i j}\right) & =\left(\begin{array}{cc}
0 & \theta(E) \\
-\theta(E) & 0
\end{array}\right), \\
\left(\eta^{i j}\right) & =\left(\begin{array}{cc}
0 & \eta(E) \\
-\eta(E) & 0
\end{array}\right), \\
\left(\Delta^{i j}\right) & =\left(\begin{array}{cc}
\gamma^{2}+\frac{\theta(E) \eta(E)}{2 \gamma^{2} \hbar^{2}} & \frac{\theta(E) \eta(E)}{4 \gamma^{2} \hbar^{2}} \\
\frac{\theta(E) \eta(E)}{4 \gamma^{2} \hbar^{2}} & \gamma^{2}+\frac{\theta(E) \eta(E)}{2 \gamma^{2} \hbar^{2}}
\end{array}\right),
\end{aligned}
$$

where $i, j$ correspond to $x, y$ and $z$. It can be seen that $\theta$ and $\eta$ are antisymmetric, but $\Delta$ is symmetric. They are assumed to be energy-dependent, and we take them as independent of the spacetime coordinates. We may set $\gamma=1$ for convenience without losing the basic physics in the following section.

Moreover, we also limit our analysis to the $x-y$ plane, where the two-dimensional noncommutative energydependent algebra can be formulated as

$[\widehat{x}, \widehat{y}]=i \theta(E),\left[\widehat{p}_{x}, \widehat{p}_{y}\right]=i \eta(E),\left[\widehat{x}_{i}, \widehat{p}_{j}\right]=i \hbar \delta_{i j}$,

$\left[E, \widehat{x}_{i}\right]=0,\left[E, \widehat{p}_{i}\right]=0, i=1,2$,

where, in the last three commutation relations, we have denoted $\widehat{x}_{1} \equiv \widehat{x}, \widehat{x}_{2} \equiv \widehat{y}, \widehat{p}_{1} \equiv \widehat{p}_{x}$ and $\widehat{p}_{2} \equiv \widehat{p}_{y}$.

Starting from the canonical quantum mechanical Heisenberg commutation relations one can easily verify that the commutation relations Eq. (18a) can be obtained through the linear transformations $[12,21]$

$$
\begin{aligned}
& \left(\begin{array}{l}
\widehat{x} \\
\widehat{y}
\end{array}\right)=\left(\begin{array}{c}
x-\frac{\theta(E)}{\hbar} p_{y} \\
y
\end{array}\right), \\
& \left(\begin{array}{c}
\widehat{p}_{x} \\
\widehat{p}_{y}
\end{array}\right)=\left(\begin{array}{c}
p_{x} \\
p_{y}-\frac{\eta(E)}{\hbar} x
\end{array}\right),
\end{aligned}
$$

or, equivalently, through the alternative set of linear transformations

$$
\begin{aligned}
& \left(\begin{array}{l}
\widehat{x} \\
\widehat{y}
\end{array}\right)=\left(\begin{array}{c}
x \\
y+\frac{\theta(E)}{\hbar} p_{x}
\end{array}\right), \\
& \left(\begin{array}{c}
\widehat{p}_{x} \\
\widehat{p}_{y}
\end{array}\right)=\left(\begin{array}{c}
p_{x}+\frac{\eta(E)}{\hbar} y \\
p_{y}
\end{array}\right) .
\end{aligned}
$$

These two types of linear transformations can be combined into a single one, which simultaneously modifies all coordinates and momenta, and not just $x$ and $p_{y}$ or $y$ and $p_{x}$, as given in Eqs. (19a), (19b) and (20a), (20b), respectively.

One possible way of implementing the algebra defined by Eqs. (18a) and (18b) is to construct the noncommutative set of variables $\left(\widehat{x}, \widehat{y}, \widehat{p}_{x}, \widehat{p}_{y}\right)$ from the commutative variables 
$\left(x, y, p_{x}, p_{y}\right)$ by means of linear transformations. This can be generally done by using the Seiberg-Witten map, given by $[12,21]$

$$
\begin{aligned}
\left(\begin{array}{l}
\widehat{x} \\
\widehat{y}
\end{array}\right) & =\left(\begin{array}{l}
x-\frac{\theta(E)}{2 \hbar} p_{y} \\
y+\frac{\theta(E)}{2 \hbar} p_{x}
\end{array}\right), \\
\left(\begin{array}{l}
\widehat{p}_{x} \\
\widehat{p}_{y}
\end{array}\right) & =\left(\begin{array}{l}
p_{x}+\frac{\eta(E)}{2 \hbar} y \\
p_{y}-\frac{\eta(E)}{2 \hbar} x
\end{array}\right),
\end{aligned}
$$

where the canonical variables $\left(x, y, p_{x}, p_{y}\right)$ satisfy Heisenberg commutation relations $[12,21]$,

$$
\begin{aligned}
{[x, y] } & =\left[p_{x}, p_{y}\right]=0, \\
{\left[x_{i}, p_{j}\right] } & =i \hbar \delta_{i j}, i=1,2,
\end{aligned}
$$

With the help of transformations (21a) and (21b) we can immediately recover the two first commutation relations in Eq. (18a). However, the last one takes the form

$$
\left[\widehat{x}_{i}, \widehat{p}_{j}\right]=i \hbar\left[1+\frac{\theta(E) \eta(E)}{4 \hbar^{2}}\right] \delta_{i j}, i=1,2 .
$$

Comparing Eqs. (18a) and (24), we find that the linear transformations given by Eqs. (21a) and (21b) generate an effective energy-dependent Planck constant, which is a function of the noncommutativity parameters $\theta(E)$ and $\eta(E)$, and it is given by [21]

$\hbar_{\mathrm{eff}}=\hbar[1+\zeta(E)]$,

where $\zeta \equiv \theta(E) \eta(E) / 4 \hbar^{2}$. This approach is consistent with the usual commutative spacetime quantum mechanics if we impose the condition $\xi \ll 1$, expected to be generally satisfied, since the small noncommutative parameters $\theta$ and $\eta, \zeta$ are of second order.

For the sake of completeness we also present the general case. In the four-dimensional spacetime equations (21a) and (21b) can be written as [21]

$$
\left(\begin{array}{l}
\widehat{x}^{\mu} \\
\widehat{p}^{\mu}
\end{array}\right)=\left(\begin{array}{c}
x^{\mu}-\frac{\theta_{\nu}^{\mu}(E)}{2 \hbar} p^{\nu} \\
p^{\mu}+\frac{\eta_{\nu}^{\mu}(E)}{2 \hbar} x^{\nu}
\end{array}\right) .
$$

Therefore we obtain the following four-dimensional commutation relations:

$$
\begin{aligned}
& {\left[\widehat{x}^{\mu}, \widehat{x}^{\nu}\right]=i \theta^{\mu \nu}(E),\left[\widehat{p}^{\mu}, \widehat{p}^{\nu}\right]=i \eta^{\mu \nu}(E),} \\
& {\left[\widehat{x}^{\mu}, \widehat{p}^{\nu}\right]=i \hbar\left[\delta^{\mu \nu}+\frac{1}{4 \hbar^{2}} \theta^{\mu \alpha}(E) \eta_{\alpha}^{\nu}(E)\right] .}
\end{aligned}
$$

Hence it follows that in the four-dimensional case the effective energy-dependent Planck constant is given by [21]

$$
\hbar_{\mathrm{eff}}=\hbar\left\{1+\frac{1}{4 \hbar^{2}} \operatorname{Tr}[\theta(E) \eta(E)]\right\} .
$$

Moreover, it also turns out that the commutator of the coordinate and momentum operators, $\left[x^{\mu}, p^{\nu}\right]$, is not diagonal any longer, with the off-diagonal elements obtained as the algebraic products of the components of $\theta^{\mu \nu}$ and $\eta^{\mu \nu}$.

The linear transformations (26) can be further generalized to the form [23]

$\widehat{x}^{\mu}=\xi\left(x^{\mu}-\frac{\theta_{\nu}^{\mu}}{2 \hbar} p^{\nu}\right), \quad \widehat{p}^{\mu}=\xi\left(p^{\mu}+\frac{\eta_{v}^{\mu}}{2 \hbar} x^{\nu}\right)$,

where $\xi$ is a scaling factor. It corresponds to a scale transformation of the coordinates and of the momenta [23]. Such a scaling can be used to make the Planck constant a true constant. Indeed, by choosing $\xi=\left(1+\theta \eta / 4 \hbar^{2}\right)^{-1 / 2}$, we obtain the two-dimensional noncommutative algebra given by [23]

$$
\begin{array}{r}
{[\widehat{x}, \widehat{y}]=i \xi^{2} \theta=i \theta_{\mathrm{eff}},\left[\widehat{p}_{x}, \widehat{p}_{y}\right]=i \xi^{2} \eta=i \eta_{\mathrm{eff}},} \\
{\left[\widehat{x}_{i}, \widehat{p}_{j}\right]=\hbar \xi^{2}\left(1+\frac{\theta \eta}{4 \hbar^{2}}\right) \delta_{i j}=i \hbar_{\mathrm{eff}} \delta_{i j}=i \hbar \delta_{i j},}
\end{array}
$$

where we have denoted

$$
\begin{aligned}
\theta_{\mathrm{eff}} & =\frac{\theta}{1+\frac{\theta \eta}{4 \hbar^{2}}}, \eta_{\mathrm{eff}}=\frac{\eta}{1+\frac{\theta \eta}{4 \hbar^{2}}}, \\
\hbar_{\mathrm{eff}} & =\hbar \xi^{2}\left(1+\frac{\theta \eta}{4 \hbar^{2}}\right)=\hbar
\end{aligned}
$$

Hence by a simple rescaling of the noncommutativity parameters one can ensure the constancy of the Planck constant. On the other hand, in [21] it was shown that by assuming that $\sqrt{\theta}$, giving the fundamental length scale in noncommutative geometry, is smaller than the average neutron size, having an order of magnitude of around $1 \mathrm{fm}$, it follows that $\left(\hbar_{\text {eff }} \hbar\right) / \hbar \leq O(10)$. Hence, in practical calculations one can ignore the deviations between the numerical values of the effective Planck constant and the usual Planck constant.

In the two-dimensional case, which we will investigate next, in order to convert a commutative Hamiltonian into a noncommutative one, we first find the inverse of the transformations given by Eqs. (21a) and (21b). This set is given by [21]

$$
\begin{aligned}
& \left(\begin{array}{l}
x \\
y
\end{array}\right)=k(E)\left(\begin{array}{l}
\widehat{x}+\frac{\theta(E)}{2 \hbar} \widehat{p}_{y} \\
\widehat{y}-\frac{\theta(E)}{2 \hbar} \widehat{p}_{x}
\end{array}\right), \\
& \left(\begin{array}{l}
p_{x} \\
p_{y}
\end{array}\right)=k(E)\left(\begin{array}{l}
\widehat{p}_{x}-\frac{\eta(E)}{2 \hbar} \widehat{y} \\
\widehat{p}_{y}+\frac{\eta(E)}{2 \hbar} \widehat{x}
\end{array}\right),
\end{aligned}
$$

where $k(E)$ is obtained:

$k(E)=\frac{1}{1-\theta(E) \eta(E) / 4 \hbar^{2}} \approx 1+\frac{\theta(E) \eta(E)}{4 \hbar^{2}}$.

In the following we will approximate $k(E)$ as being one, $k(E) \approx 1$. 


\section{The Schrödinger equation in the energy-dependent noncommutative geometry}

In order to develop some basic physics applications in the energy-dependent noncommutative quantum mechanics, as a first step we investigate a 2-D noncommutative quantum system by using the map between the energy-dependent noncommutative algebra and the Heisenberg algebra (21a) and (21b). In this approach the Hamiltonian $\widehat{H}$ of a particle in an exterior potential $V$ can be obtained:

$$
\begin{aligned}
\widehat{H}= & \frac{1}{2 m}\left\{\left[p_{x}-\frac{\eta(E)}{2 \hbar} y\right]^{2}+\left[p_{y}+\frac{\eta(E)}{2 \hbar} x\right]^{2}\right\} \\
& +V\left[\left(x+\frac{\theta(E)}{2 \hbar} p_{y}\right),\left(y-\frac{\theta(E)}{2 \hbar} p_{x}\right)\right] .
\end{aligned}
$$

Equivalently, the two-dimensional Hamiltonian (32) can be written as

$$
\begin{aligned}
\widehat{H}= & \frac{1}{2 m}\left(p_{x}^{2}+p_{y}^{2}\right)+\frac{\eta(E)}{2 m \hbar}\left(x p_{y}-y p_{x}\right)+\frac{\eta^{2}(E)}{8 m \hbar^{2}} \\
& \times\left(x^{2}+y^{2}\right)+V\left[\left(x+\frac{\theta(E)}{2 \hbar} p_{y}\right),\left(y-\frac{\theta(E)}{2 \hbar} p_{x}\right)\right] .
\end{aligned}
$$

Consequently, we obtain the generalized Schrödinger equation in the noncommutative geometry with energydependent strengths:

$i \hbar \frac{\partial}{\partial t} \Psi(x, y, t)=\widehat{H} \Psi(x, y, t)$,

where the total Hamiltonian $H$ can be written as

$\widehat{H}=H_{0}+V_{\text {eff }}$,

with

$H_{0}=\frac{1}{2 m}\left(p_{x}^{2}+p_{y}^{2}\right)$

is the standard quantum mechanical kinetic energy in the Heisenberg representation. In general, the effective potential $V_{\text {eff }}$ in Eq. (35) is given by

$$
\begin{aligned}
V_{\mathrm{eff}}\left(x, y, p_{x}, p_{y}\right)= & \frac{\eta(E)}{2 m \hbar}\left(x p_{y}-y p_{x}\right)+\frac{\eta^{2}(E)}{8 m \hbar^{2}}\left(x^{2}+y^{2}\right) \\
& +V\left[\left(x+\frac{\theta(E)}{2 \hbar} p_{y}\right),\left(y-\frac{\theta(E)}{2 \hbar} p_{x}\right)\right]
\end{aligned}
$$

it comes from both of the kinetic energy and the potential in the noncommutative algebra.
3.1 Probability current and density in the energy-dependent potential

One of the interesting properties of the energy-dependent Schrödinger equation is that it leads to modified versions of the probability density and of the probability current [6264]. This also implies modifications in the scalar product and the norm of the vectors in the Hilbert space. To investigate the nature of these modifications we will consider the onedimensional Schrödinger equation in an energy-dependent potential $V=V(x, y, E)$, which is given by

$i \hbar \frac{\partial \Psi(x, y, t)}{\partial t}=\left[-\frac{\hbar^{2}}{2 m} \Delta_{2}+V(x, y, E)\right] \Psi(x, y, t)$,

where $\Delta_{2}=\nabla_{2} \cdot \nabla_{2}=\left(\frac{\partial^{2}}{\partial x^{2}}+\frac{\partial^{2}}{\partial y^{2}}\right)$, with $\nabla_{2}=\frac{\partial}{\partial x} \vec{i}+\frac{\partial}{\partial y} \vec{j}$.

Let us now consider two solutions of energy $E$ and $E^{\prime}$ of the above Schrödinger equation, given by

$\Phi_{\epsilon}(x, y, t)=\mathrm{e}^{-\frac{i}{\hbar}(E-i \epsilon) t} \Phi(x, y)$,

$\Psi_{\epsilon}(x, y, t)=\mathrm{e}^{-\frac{i}{\hbar}\left(E^{\prime}-i \epsilon\right) t} \Psi(x, y)$,

where $\epsilon$ is a small parameter, $\epsilon \rightarrow 0$. Then from the Schrödinger equation (38) we obtain the continuity equation:

$\frac{\partial \rho}{\partial t}+\nabla \cdot \mathbf{J}=0$

where

$$
\begin{aligned}
\rho= & \Psi_{\epsilon}^{*}(x, y, t) \Phi_{\epsilon}(x, y, t)+\rho_{a}(x, y, t), \\
\mathbf{J}= & -\frac{\hbar^{2}}{2 i m}\left[\Psi_{\epsilon}^{*}(x, y, t) \nabla_{2} \Phi_{\epsilon}(x, y, t)\right. \\
& \left.-\Phi_{\epsilon}(x, t) \nabla_{2} \Psi^{*}(x, t)\right],
\end{aligned}
$$

and $\rho_{a}$ is obtained as a solution of the equation

$$
\frac{\partial}{\partial t} \rho_{a}=\frac{i}{\hbar} \Psi^{*}(x, y, t)\left[V(x, y, E)-V\left(x, y, E^{\prime}\right)\right] \Phi_{\epsilon}(x, y, t) .
$$

By taking into account the explicit form of the wave functions as given in Eqs. (39) and (40), after integration and taking the limit $\epsilon \rightarrow 0$, we obtain for $\rho_{a}$ the expression

$\rho_{a}(x, y)=-\Psi^{*}(x, y)\left[\frac{V\left(x, y, E^{\prime}\right)-V(x, y, E)}{\left(E^{\prime}-E\right)}\right] \Phi(x, y)$. 
By considering limit $E^{\prime} \rightarrow E$ it follows that for the energydependent wave function its norm (scalar product) in the Hilbert space is defined as [62]

$N=\int_{-\infty}^{+\infty} \Psi^{*}(x, y)\left[1-\frac{\partial V(x, y, E)}{\partial E}\right] \Psi(x, y) \mathrm{d} x \mathrm{~d} y>0$.

If we specify the stationary states by their quantum numbers $n$, it follows that the orthogonality relation between two states $n$ and $n^{\prime}, n \neq n^{\prime}$, is given by

$$
\int \Psi_{n^{\prime}}^{*}(x, y)\left[1-\varphi_{n^{\prime} n}(x, y)\right] \Psi_{n}(x, y) \mathrm{d} x \mathrm{~d} y=0,
$$

where

$\varphi_{n^{\prime} n}(x, y)=\frac{V\left(x, y, E_{n^{\prime}}\right)-V\left(x, y, E_{n}\right)}{\left(E_{n^{\prime}}-E_{n}\right)}$.

In the case of the energy-dependent quantum mechanical systems the standard completeness relation $\sum_{n} \Psi_{n}\left(x^{\prime}, y^{\prime}\right)$ $\Psi_{n}^{*}(x, y)=\delta\left(x-x^{\prime}\right) \delta\left(y-y^{\prime}\right)$ does not hold generally. This is a consequence of the fact that the functions $\Psi_{n}(x, y)$ do not represent eigenfunctions of the same (linear self-adjoint) operator on $L^{2}(-\infty,+\infty)$. An alternative procedure was proposed in [62], and it is given by $\sum_{n} \Psi_{n}\left(x^{\prime}, y^{\prime}\right)\left[1-\phi_{n n^{\prime}}(x, y)\right] \Psi_{n}^{*}(x, y)=\delta\left(x-x^{\prime}\right) \delta$ $\left(y-y^{\prime}\right)$. Finally, we would like to mention that due to the presence of the energy eigenvalue in the Hamiltonian the commutator $[H, x]$ is obtained as $[H, x]=-i \hbar p+$ $[\partial V(H, x) / \partial H](\partial H / \partial p)$, and similarly for the coordinate $y$.

\subsection{The free particle}

For free particles, $V(\widehat{x}, \widehat{y})=0$, and the effective Hamiltonian takes the form

$\widehat{H}=\frac{1}{2 m}\left(p_{x}^{2}+p_{y}^{2}\right)+V_{\text {eff }}\left(x, y, p_{x}, p_{y}\right)$,

where the effective potential comes from the kinetic energy term only via the Seiberg-Witten map, and it is given by

$$
\begin{aligned}
V_{\mathrm{eff}}\left(x, y, p_{x}, p_{y}\right) & =-\frac{\eta(E)}{2 m \hbar} L_{z}+\frac{\eta^{2}(E)}{8 m \hbar^{2}}\left(x^{2}+y^{2}\right) \\
& \equiv-B_{e}(E) L_{z}+\frac{k_{e}(E)}{2}\left(x^{2}+y^{2}\right),
\end{aligned}
$$

where $L_{z}=\left(x p_{y}-y p_{x}\right)$ is the $z$-component of the angular momentum,

$B_{e}(E)=\frac{\eta(E)}{2 m \hbar}$, can be interpreted as an effective magnetic field, while

$k_{e}(E)=\frac{\eta^{2}(E)}{8 m \hbar^{2}}$,

is the effective elastic constant corresponding to a harmonic oscillator. Hence the effective potential for free particles induced by the noncommutative algebra can be interpreted as generating two distinct physical processes: an effective magnetic field and an effective harmonic oscillator, respectively.

\subsection{The harmonic oscillator}

As a second example of quantum evolution in the noncommutative geometry with energy-dependent noncommutative strengths let us consider the case of the two-dimensional quantum harmonic oscillator. The potential energy is written as

$V(\widehat{x}, \widehat{y})=\frac{1}{2} k\left(\widehat{x}^{2}+\widehat{y}^{2}\right)$,

where $k$ is a constant. By using the 2D Seiberg-Witten map as given by Eq. (21a), the potential can be expressed as

$$
\begin{aligned}
& V\left[\left(x+\frac{\theta(E)}{2 \hbar} p_{y}\right),\left(y-\frac{\theta(E)}{2 \hbar} p_{x}\right)\right] \\
& \quad=V(x, y)+\frac{k}{2}\left[-\frac{\theta(E)}{\hbar} L_{z}+\frac{\theta^{2}(E)}{4 \hbar^{2}}\left(p_{x}^{2}+p_{y}^{2}\right)\right],
\end{aligned}
$$

where $V(x, y)=\frac{1}{2} k\left(x^{2}+y^{2}\right)$ is the potential energy of the harmonic oscillator in Heisenberg's representation. The Hamiltonian can be written as

$\widehat{H}=\frac{1}{2 m^{*}}\left(p_{x}^{2}+p_{y}^{2}\right)-B_{h} L_{z}+\frac{1}{2} K_{h}\left(x^{2}+y^{2}\right)$,

where

$$
\begin{aligned}
& \frac{1}{m^{*}}=\frac{1}{m}+\frac{k}{4 \hbar^{2}} \theta^{2}(E), \\
& B_{h}(E)=B_{e}(E)+\frac{k \theta(E)}{2 \hbar}=\frac{\eta(E)}{2 m \hbar}+\frac{k \theta(E)}{2 \hbar}, \\
& K_{h}(E)=k+k_{e}(E)=k+\frac{\eta^{2}(E)}{8 m \hbar^{2}} .
\end{aligned}
$$

In the above equations $m^{*}$ is the effective mass of the oscillator, including the modifications of the harmonic potential due to the noncommutative algebra, $B_{h}$ is the effective magnetic field, in which the first term comes from the kinetic energy and the second term comes from the harmonic potential energy in the noncommutative algebra, while $K_{h}$ is the 
effective elastic constant, in which the second term comes from the noncommutative algebra. For free particle, namely $V(\widehat{x}, \widehat{y})=0, m^{*}=m, B_{h}=B_{e}(E)$, and $K_{h}=k_{e}(E)$. The Hamiltonian (55) gives a unified description of the quantum evolution for both the free particle and for the harmonic oscillator in the energy-dependent noncommutative geometry. By taking $k=0$ we immediately obtain the case of the free particle.

\section{Physical mechanisms generating energy-dependent noncommutative algebras, and their implications}

The idea of the energy-dependent noncommutative geometry and its underlying algebra must be supplemented by the description of different physical processes that could lead to such mathematical structures. In the following we propose several possible mechanisms that could generate quantum energy-dependent behaviors described by the corresponding noncommutative algebra.

- We assume first that there exists an intrinsic and universal energy scale $\varepsilon$, different of the particle energy scale $E$, which induces the noncommutative effects, and the corresponding algebra. This intrinsic universal energy scale could be related to the spacetime quantum fluctuations (SQF), and to the Planck energy scale, respectively. Therefore the energy-dependence in the commutation relations is determined by the $\epsilon$ energy scale, or by the magnitude of the quantum fluctuations. Hence in this approach the dynamics of the quantum particle is determined by two independent energy scales.

- For the second mechanism we assume that there is an energy coupling (EC) between the noncommutative evolution, and the dynamical energy $E$ of the quantum systems. Hence in this approach the interaction between the particle dynamics and the spacetime fluctuations is fully determined by the particle energy, and all physical processes related to the energy-dependent noncommutativity are described in terms of the particle energy scale $E$.

- Finally, the third mechanism we are going to consider follows from the possibility that the energy of a quantum system can be mapped to an energy operator, which modifies the Hamiltonian of the system, and the corresponding Schrödinger equation. This approach we call the EO (energy operator) approach; it assumes again that the dominant energy scale describing noncommutative effects is the particle energy scale, $E$.

In the following we will consider in detail the mathematical formulations of the above physical mechanisms, and their physical implications.
4.1 The noncommutative algebra of the spacetime quantum fluctuations (SQF) model

Let us consider first there exists an intrinsic and universal energy scale $\varepsilon$ inducing the noncommutative algebra. This energy scale is different and independent from the particle energy $E$. Hence in this approach we are dealing with a model with two distinct energy scales. For the sake of concreteness we assume that the noncommutativity parameters $\eta$ and $\theta$ have a power-law dependence on the intrinsic energy scale $\varepsilon$, so that

$\eta(\varepsilon)=\eta_{0}\left(\frac{\varepsilon}{\varepsilon_{0}}\right)^{\alpha}, \quad \theta(\varepsilon)=\theta_{0}\left(\frac{\varepsilon}{\varepsilon_{0}}\right)^{\beta}$,

with $\eta_{0}, \theta_{0}, \alpha, \beta$ are parameters describing the strength of the energy-dependent noncommutative effects. The energy parameter $\varepsilon_{0}$ describes the basic energy scale, which is related to the spacetime quantum fluctuation or Planck scale. This energy-dependent noncommutative mechanism is called the spacetime quantum fluctuation ( $\mathrm{SQF}$ ) process. When $\varepsilon \ll \varepsilon_{0}$, both $\eta(\varepsilon)$ and $\theta(\varepsilon)$ tend to zero, and thus we recover the canonical quantum mechanics. When $\varepsilon \approx$ $\varepsilon_{0}$, we reach the opposite limit of noncommutative quantum mechanics with constant noncommutative parameters. Thus, the basic physical parameters describing of effects of the noncommutativity in the effective potential of the Schrödinger equation in the framework of the power-law energy-dependent strength noncommutative algebra Eq. (50) become

$$
\begin{aligned}
& B_{e}(E) \rightarrow B_{\varepsilon}=\frac{\eta_{0}}{2 m \hbar}\left(\frac{\varepsilon}{\varepsilon_{0}}\right)^{\alpha} \equiv B_{0}\left(\frac{\varepsilon}{\varepsilon_{0}}\right)^{\alpha}, \\
& k_{e}(E) \rightarrow k_{\varepsilon}=\frac{\eta_{0}^{2}}{8 m \hbar^{2}}\left(\frac{\varepsilon}{\varepsilon_{0}}\right)^{2 \alpha} \equiv \frac{k_{0}}{2}\left(\frac{\varepsilon}{\varepsilon_{0}}\right)^{2 \alpha},
\end{aligned}
$$

where $B_{0}=\frac{\eta_{0}}{2 m \hbar}$ and $k_{0}=\frac{\eta_{0}^{2}}{4 m \hbar^{2}}$. For the harmonic oscillator we obtain

$$
\begin{aligned}
& \frac{1}{m^{*}}=\frac{1}{m}+\frac{k}{4 \hbar^{2}} \theta_{0}^{2}\left(\frac{\epsilon}{\epsilon_{0}}\right)^{2 \beta} \\
& B_{h}(\epsilon)=\frac{\eta_{0}}{2 m \hbar}\left(\frac{\epsilon}{\epsilon_{0}}\right)^{\alpha}+\frac{k}{2 \hbar} \theta_{0}\left(\frac{\epsilon}{\epsilon_{0}}\right)^{\beta}
\end{aligned}
$$

and

$K_{h}(\epsilon)=k+\frac{\eta_{0}^{2}}{8 m \hbar^{2}}\left(\frac{\epsilon}{\epsilon_{0}}\right)^{2 \alpha}$,

respectively. 
4.2 The noncommutative algebra of the energy coupling (EC) model

In our second model we assume that there is a coupling between the energy-dependent noncommutative geometry, and the energy of the quantum dynamical systems, and that this coupling can be described in terms of the particle energy $E$ only. By adopting again a power-law dependence of the noncommutativity parameters $\eta$ and $\theta$ on the particle energy $E$ we have

$\eta(E)=\eta_{0}\left(\frac{E}{E_{0}}\right)^{\alpha}, \quad \theta(E)=\theta_{0}\left(\frac{E}{E_{0}}\right)^{\beta}$,

with $\eta_{0}, \theta_{0}, \alpha, \beta$ are parameters describing the strength of the noncommutative effects. $E_{0}$ is a critical energy, which can interpreted as the ground-state energy of the quantum system, or a critical energy in some phase transition. We call this energy-dependent noncommutativity generating mechanism the energy coupling (EC) mechanism. When $E \ll E_{0}$, both $\eta(E)$ and $\theta(E)$ tend to zero, and the noncommutative algebra reduces to the standard Heisenberg algebra. When $E \approx E_{0}$, we reach the opposite limit of noncommutative quantum mechanics with constant parameters. Similarly, the physical parameters of the power-law energy-dependent noncommutative algebra in the effective potential given by Eq. (50) take the form

$B_{e}(E)=\frac{\eta_{0}}{2 m \hbar}\left(\frac{E}{E_{0}}\right)^{\alpha} \equiv B_{0}\left(\frac{E}{E_{0}}\right)^{\alpha}$,

$k_{e}(E)=\frac{\eta_{0}^{2}}{8 m \hbar^{2}}\left(\frac{E}{E_{0}}\right)^{2 \alpha} \equiv \frac{k_{0}}{2}\left(\frac{E}{E_{0}}\right)^{2 \alpha}$,

where $B_{0}=\frac{\eta_{0}}{2 m \hbar}$ and $k_{0}=\frac{\eta_{0}^{2}}{4 m \hbar^{2}}$.

4.3 The noncommutative algebra of the energy operator (EO) model

Finally, we consider the model in which the energy-dependent noncommutative geometry can be mapped to a quantum mechanical representation. This can be realized by associating a quantum operator to the considered energy scales $\epsilon$ or $E$. There are two possibilities to construct such a mapping between energy and operators.

Case I. In the first case we consider the mapping $\varepsilon \rightarrow i \hbar \frac{\partial}{\partial t}$, that is, we map the energy to the standard quantum mechanical representation. Hence we obtain for the noncommutativity parameters the representation

$$
\begin{aligned}
& \eta(\varepsilon)=\eta_{0}\left(\frac{\varepsilon}{\varepsilon_{0}}\right)^{\alpha} \rightarrow \eta_{0}\left(\frac{i \hbar}{\varepsilon_{0}}\right)^{\alpha}\left(\frac{\partial}{\partial t}\right)^{\alpha} \equiv \eta_{\alpha}^{I} D_{t}^{\alpha}, \\
& \theta(\varepsilon)=\theta_{0}\left(\frac{\varepsilon}{\varepsilon_{0}}\right)^{\beta} \rightarrow \theta_{0}\left(\frac{i \hbar}{\varepsilon_{0}}\right)^{\beta}\left(\frac{\partial}{\partial t}\right)^{\beta} \equiv \theta_{\beta}^{I} D_{t}^{\beta},
\end{aligned}
$$

where

$\eta_{\alpha}^{I}=\eta_{0}\left(\frac{i \hbar}{\varepsilon_{0}}\right)^{\alpha}, \quad \theta_{\beta}^{I}=\theta_{0}\left(\frac{i \hbar}{\varepsilon_{0}}\right)^{\beta}$.

The effective magnetic field and elastic constant in Eq. (61) for Case I are represented by operators that can be expressed in terms of fractional derivative as [71-73]

$B_{e}(E) \rightarrow B_{0}\left(\frac{i \hbar}{\varepsilon_{0}}\right)^{\alpha}\left(\frac{\partial}{\partial t}\right)^{\alpha} \equiv B_{\alpha}^{I} D_{t}^{\alpha}$,
$k_{e}(E) \rightarrow \frac{k_{0}}{2}\left(\frac{i \hbar}{\varepsilon_{0}}\right)^{2 \alpha}\left(\frac{\partial}{\partial t}\right)^{2 \alpha} \equiv \frac{k_{\alpha}^{I}}{2} D_{t}^{\alpha}$,

where $B_{\alpha}^{I} \equiv B_{0}\left(\frac{i \hbar}{\varepsilon_{0}}\right)^{\alpha}, k_{\alpha}^{I} \equiv k_{0}\left(\frac{i \hbar}{\varepsilon_{0}}\right)^{2 \alpha}$ and $D_{t}^{\alpha} \equiv\left(\frac{\partial}{\partial t}\right)^{\alpha}$.

Case II. In the second case we assume that the energy can be mapped to the Hamiltonian operator according to the rule

$\varepsilon \rightarrow H=-\frac{\hbar^{2}}{2 m} \triangle_{2}$

In this case for the power-law dependent noncommutativity parameters we obtain

$\eta(\varepsilon)=\eta_{0}\left(\frac{\varepsilon}{\varepsilon_{0}}\right)^{\alpha} \rightarrow \eta_{0}\left(\frac{-\hbar^{2}}{2 m \varepsilon_{0}}\right)^{\alpha}\left(\Delta_{2}\right)^{\alpha} \equiv \eta_{\alpha}^{I I} \Delta_{2}^{\alpha}$,
$\theta(\varepsilon)=\theta_{0}\left(\frac{\varepsilon}{\varepsilon_{0}}\right)^{\beta} \rightarrow \theta_{0}\left(\frac{-\hbar^{2}}{2 m \varepsilon_{0}}\right)^{\beta}\left(\triangle_{2}\right)^{\beta} \equiv \theta_{\beta}^{I I} \Delta_{2}^{\beta}$,

where

$\eta_{\alpha}^{I I}=\eta_{0}\left(\frac{-\hbar^{2}}{2 m \varepsilon_{0}}\right)^{\alpha}, \theta_{\beta}^{I I}=\theta_{0}\left(\frac{-\hbar^{2}}{2 m \varepsilon_{0}}\right)^{\beta}$

The effective magnetic field and the elastic constant in Eq. (65) are represented by the fractional derivatives,

$$
\begin{aligned}
& B_{e}(E) \rightarrow B_{0}\left(\frac{-\hbar^{2}}{2 m \varepsilon_{0}}\right)^{\alpha}\left(\triangle_{2}\right)^{\alpha} \equiv B_{\alpha}^{I I}\left(\triangle_{2}\right)^{\alpha}, \\
& k_{e}(E) \rightarrow \frac{k_{0}}{2}\left(\frac{-\hbar^{2}}{2 m \varepsilon_{0}}\right)^{2 \alpha}\left(\triangle_{2}\right)^{2 \alpha} \equiv \frac{k_{\alpha}^{I I}}{2}\left(\triangle_{2}\right)^{2 \alpha},
\end{aligned}
$$

where $B_{\alpha}^{I I} \equiv B_{0}\left(\frac{-\hbar^{2}}{2 m \varepsilon_{0}}\right)^{\alpha}, k_{\alpha}^{I I} \equiv k_{0}\left(\frac{-\hbar^{2}}{2 m \varepsilon_{0}}\right)^{2 \alpha}$, and $\left(\triangle_{2}\right)^{\alpha} \equiv\left(\frac{\partial^{2}}{\partial x^{2}}+\frac{\partial^{2}}{\partial y^{2}}\right)^{\alpha}$, respectively.

Since $\alpha, \beta$ are real variables, the energy-dependent noncommutative geometry in the EO model now involves fractional derivative differential equations.

Hence the energy operator (EO) representation of the energy-dependent noncommutative quantum mechanics leads to the emergence of fractional calculus for the physical description of the high-energy scale quantum processes. In general there are several definitions of the fractional derivatives, which we will discuss briefly in Sect. 7. 
For convenience we rewrite the notations of Cases I and II in a unified form,

$$
\begin{aligned}
& B_{e}(E) \rightarrow B_{\alpha}^{\ell} \mathcal{D}_{\ell}^{\alpha}, \\
& k_{e}(E) \rightarrow \frac{k_{\alpha}^{\ell}}{2} \mathcal{D}_{\ell}^{\alpha},
\end{aligned}
$$

where $\ell=I, I I, \mathcal{D}_{I}^{\alpha}=D_{t}^{\alpha}$ and $\mathcal{D}_{I I}^{\alpha}=\Delta_{2}^{\alpha}$ for Cases I and II.

\section{Quantum evolution in the spacetime quantum fluctuation (SQF) energy-dependent noncommutative model}

In the present section we explore the physical implications of the SQF noncommutative algebra and the underlying quantum evolution. To gain some insights into the effects of the energy-dependent noncommutativity on the dynamics of quantum particles we analyze two basic models of quantum mechanics - the free particle and the harmonic oscillator, respectively.

\subsection{Quantum mechanics of the free particle in the SQF model}

Let first us consider a free particle whose quantum mechanical evolution is described by the SQF noncommutative algebra with $V(\widehat{x}, \widehat{y})=0$. Hence the effective potential becomes

$V_{\mathrm{eff}}=-B_{\varepsilon} L_{z}+\frac{k_{\varepsilon}}{2}\left(x^{2}+y^{2}\right)$.

The generalized Schrödinger equation reduces to

$i \hbar \frac{\partial}{\partial t} \Psi(x, y, t)=\widehat{H} \Psi(x, y, t)$,

where

$\widehat{H}=-\frac{\hbar^{2}}{2 m} \triangle_{2}-B_{\varepsilon} L_{z}+\frac{k_{\varepsilon}}{2}\left(x^{2}+y^{2}\right)$,

and with $B_{\varepsilon}$ and $k_{\varepsilon}$ given by Eqs. (58a) and (58b), respectively. Since $H_{0}$ is independent of time, the wave function is of the form

$\Psi(t, x, y)=\mathrm{e}^{-\frac{i}{\hbar} E t} \psi(x, y)$

where $E$ is the energy of the free particle. By substituting the wave function (74) into the Schrödinger equation (72), the stationary Schrödinger equation is obtained:

$$
\left[-\frac{\hbar^{2}}{2 m} \triangle_{2}-B_{\varepsilon} L_{z}+\frac{k_{\varepsilon}}{2}\left(x^{2}+y^{2}\right)\right] \psi(x, y)=E \psi(x, y) .
$$

We now introduce the particle representation,

$$
\begin{aligned}
& \left(\begin{array}{c}
\widehat{a} \\
\widehat{a}^{\dagger}
\end{array}\right)=\sqrt{\frac{m \omega_{\varepsilon}}{2 \hbar}}\left(\begin{array}{l}
x-\frac{1}{i \hbar m \omega_{\varepsilon}} \frac{\partial}{\partial x} \\
x+\frac{1}{i \hbar m \omega_{\varepsilon}} \frac{\partial}{\partial x}
\end{array}\right), \\
& \left(\begin{array}{c}
\widehat{b} \\
\widehat{b}^{\dagger}
\end{array}\right)=\sqrt{\frac{m \omega_{\varepsilon}}{2 \hbar}}\left(\begin{array}{l}
y-\frac{1}{i \hbar m \omega_{\varepsilon}} \frac{\partial}{\partial y} \\
y+\frac{1}{i \hbar m \omega_{\varepsilon}} \frac{\partial}{\partial y}
\end{array}\right),
\end{aligned}
$$

where $\widehat{a}$ and $\widehat{b}^{\dagger}$ are the particle annihilation and creation operators, and we have denoted $\omega_{\varepsilon}=\sqrt{\frac{k_{\varepsilon}}{m}}$. Then it is easy to show that the operators $\widehat{a}$ and $\widehat{b}^{\dagger}$ satisfy the Bose algebra, namely $\left[\widehat{a}, \widehat{a}^{\dagger}\right]=1$ and $\left[\widehat{b}, \widehat{b}^{\dagger}\right]=1$, respectively. The other operators are commutative. Hence the Hamiltonian can be represented as

$\widehat{H}_{0}=\left(\widehat{a}^{\dagger} \widehat{b}\right)\left(\begin{array}{cc}\hbar \omega_{\varepsilon} & i \hbar B_{\varepsilon} \\ -i \hbar B_{\varepsilon} & \hbar \omega_{\varepsilon}\end{array}\right)\left(\begin{array}{c}\widehat{a} \\ \widehat{b}^{\dagger}\end{array}\right)$

By using the Bogoliubov transformation,

$\left(\begin{array}{c}\widehat{\alpha}^{\dagger} \\ \widehat{\beta}\end{array}\right)=\left(\begin{array}{ll}u & v \\ v & u\end{array}\right)\left(\begin{array}{c}\widehat{a} \\ \widehat{b}^{\dagger}\end{array}\right)$

to diagonalize the Hamiltonian, we obtain

$\widehat{H}_{0}=\hbar \Omega_{\alpha}\left(\widehat{\alpha}^{\dagger} \widehat{\alpha}+\widehat{\beta}^{\dagger} \widehat{\beta}+1\right)$

where

$\Omega_{\alpha}=\omega_{\varepsilon}+B_{\varepsilon}=\sqrt{\frac{k_{\varepsilon}}{m}}+B_{\varepsilon}=\frac{\eta_{0}}{2 m \hbar}\left(1+\frac{1}{\sqrt{2}}\right)\left(\frac{\varepsilon}{\varepsilon_{0}}\right)^{\alpha}$

is the effective frequency of the effective "harmonic oscillator" associated with the quantum evolution of the free particle. The eigenvalues of $\widehat{H}_{0}$ can be written as

$E=\hbar \Omega_{\alpha}\left(n_{\alpha}+n_{\beta}+1\right)$

where $n_{\alpha}, n_{\beta}=0,1, \ldots$ The corresponding eigenstates can be expressed as

$\left|\psi_{n_{\alpha}, n_{\beta}}\right\rangle=\frac{1}{\sqrt{n_{\alpha} ! n_{\beta} !}} \widehat{n}_{\alpha} \widehat{n}_{\beta}|0,0\rangle$

where $\widehat{n}_{\alpha}=\widehat{\alpha}^{\dagger} \widehat{\alpha}$ and $\widehat{n}_{\beta}=\widehat{\beta}^{\dagger} \widehat{\beta}$ are the quasi- particle operators, and $|0,0\rangle$ is the ground state of the associated two-dimensional harmonic oscillator. 


\subsection{The harmonic oscillator}

For the two-dimensional quantum harmonic oscillator in the SQF noncommutative geometry, the potential energy by using the 2D Seiberg-Witten map as given by Eq. (21a) can be expressed as

$V(\widehat{x}, \widehat{y})=\frac{1}{2} k\left(\widehat{x}^{2}+\widehat{y}^{2}\right)$,

giving

$$
\begin{gathered}
V\left[\left(x+\frac{\theta(E)}{2 \hbar} p_{y}\right),\left(y-\frac{\theta(E)}{2 \hbar} p_{x}\right)\right] \\
=V(x, y)+\frac{k}{2}\left[-\frac{\theta_{0}}{\hbar}\left(\frac{\varepsilon}{\varepsilon_{0}}\right)^{\alpha} L_{z}\right. \\
\left.+\frac{\theta_{0}^{2}}{4 \hbar^{2}}\left(\frac{\varepsilon}{\varepsilon_{0}}\right)^{2 \beta}\left(p_{x}^{2}+p_{y}^{2}\right)\right],
\end{gathered}
$$

where $V(x, y)=\frac{1}{2} k\left(x^{2}+y^{2}\right)$ is the potential energy of the harmonic oscillator in the Heisenberg representation. The generalized Schrödinger equation takes the form

$i \hbar \frac{\partial}{\partial t} \Psi(x, y, t)=\widehat{H} \Psi(x, y, t)$,

where

$\widehat{H}=-\frac{\hbar^{2}}{2 m^{*}} \triangle_{2}-B_{h} L_{z}+\frac{1}{2} K_{h}\left(x^{2}+y^{2}\right)$,

and we have denoted

$$
\begin{aligned}
& \frac{1}{m^{*}}=\frac{1}{m}+\frac{2 \kappa_{\varepsilon}}{\hbar^{2}}, \quad \kappa_{\varepsilon}=\frac{k \theta_{0}^{2}}{8}\left(\frac{\varepsilon}{\varepsilon_{0}}\right)^{2 \beta}, \\
& B_{h}=B_{\varepsilon}+\frac{k \theta_{0}}{2 \hbar}\left(\frac{\varepsilon}{\varepsilon_{0}}\right)^{\beta}, K_{h}=k+k_{0}\left(\frac{\varepsilon}{\varepsilon_{0}}\right)^{2 \beta} .
\end{aligned}
$$

In the above equations $m^{*}$ is the effective mass of the oscillator, including the modifications of the harmonic potential due to the SQF noncommutative algebra, $B_{h}$ is the effective magnetic field, in which the first term comes from the kinetic energy and the second term comes from the potential energy in the SQF noncommutative algebra, while $K_{h}$ is the effective elastic constant, in which the second term comes from the SQF noncommutative algebra. For a free particle, namely $V(\widehat{x}, \widehat{y})=0, m^{*}=m, B_{h}=B_{\varepsilon}$, and $K_{h}=k_{0}\left(\frac{\varepsilon}{\varepsilon_{0}}\right)^{2 \alpha}$.

Similarly to the free particle case, since $H$ is independent of time, the wave function is of the form

$\Psi(t, x, y)=\mathrm{e}^{-\frac{i}{\hbar} E t} \psi(x, y)$, where $E$ is the energy of the oscillator. By substituting the wave function (88) into the Schrödinger equation (85), we obtain the stationary Schrödinger equation as given by

$$
\left[-\frac{\hbar^{2}}{2 m^{*}} \triangle_{2}-B_{h} L_{z}+\frac{K_{h}}{2}\left(x^{2}+y^{2}\right)\right] \psi(x, y)=E \psi(x, y) .
$$

By using the same procedure as in the case of the free particle, we diagonalize the Hamiltonian, thus obtaining

$\widehat{H}=\hbar \Omega_{\alpha}\left(\widehat{\alpha}^{\dagger} \widehat{\alpha}+\widehat{\beta}^{\dagger} \widehat{\beta}+1\right)$,

where

$$
\begin{aligned}
\Omega_{\alpha}= & \omega_{h}+B_{h}=\sqrt{\frac{k}{m^{*}}+\frac{k_{0}}{m^{*}}\left(\frac{\varepsilon}{\varepsilon_{0}}\right)^{2 \alpha}} \\
& +\frac{\eta_{0}}{2 m \hbar}\left(\frac{\epsilon}{\epsilon_{0}}\right)^{\alpha}\left[1+\frac{k m \theta_{0}}{\eta_{0}}\left(\frac{\epsilon}{\epsilon_{0}}\right)^{\beta-\alpha}\right],
\end{aligned}
$$

where $\omega_{h}=\sqrt{K_{h} / m^{*}}$ is the generalized effective frequency of the two-dimensional harmonic oscillator in the SQF noncommutative algebra. The eigenvalues of $\widehat{H}$ can be written as

$E=\hbar \Omega_{\alpha}\left(n_{\alpha}+n_{\beta}+1\right)$,

where $n_{\alpha}, n_{\beta}=0,1, \ldots$ The corresponding eigenstates can be obtained:

$\left|\psi_{n_{\alpha}, n_{\beta}}\right\rangle=\frac{1}{\sqrt{n_{\alpha} ! n_{\beta} !}} \widehat{n}_{\alpha} \widehat{n}_{\beta}|0,0\rangle$,

where $\widehat{n}_{\alpha}=\widehat{\alpha}^{\dagger} \widehat{\alpha}$ and $\widehat{n}_{\beta}=\widehat{\beta}^{\dagger} \widehat{\beta}$ are the quasi-particle operators, and $|0,0\rangle$ is the ground state of the two-dimensional harmonic oscillator in the SQF noncommutative algebra.

\section{Quantum dynamics in the energy coupling model}

In the present section we investigate the two basic quantum mechanical models, the free particle, and the harmonic oscillator, respectively, in the energy coupling (EC) noncommutative algebra, by assuming that the noncommutativity parameters $\theta$ and $\eta$ are functions of the particle energy $E$ only, and independent of energy scale of the quantum spacetime fluctuations.

\subsection{Quantum evolution: the Schrödinger equation}

For the EC noncommutative algebra, the effective potential can be written in a unified form for both the free particle and the harmonic potential: 
$V_{\mathrm{eff}}=-B_{h}(E) L_{z}+\frac{K_{h}(E)}{2}\left(x^{2}+y^{2}\right)$.

Then the generalized Schrödinger equation can be obtained:

$i \hbar \frac{\partial}{\partial t} \Psi(x, y, t)=\widehat{H} \Psi(x, y, t)$,

where

$\widehat{H}=-\frac{\hbar^{2}}{2 m^{*}} \triangle_{2}-B_{h}(E) L_{z}+\frac{K_{h}(E)}{2}\left(x^{2}+y^{2}\right)$.

Since $\widehat{H}$ is independent of time, the wave function is of the form

$\Psi(t, x, y)=\mathrm{e}^{-\frac{i}{\hbar} E t} \psi(x, y)$,

By substituting the wave function (97) into the Schrödinger equation (95), we obtain the stationary Schrödinger equation as

$\left[-\frac{\hbar^{2}}{2 m^{*}} \triangle_{2}-B_{h}(E) L_{z}+\frac{K_{h}(E)}{2}\left(x^{2}+y^{2}\right)\right] \psi=E \psi$.

\subsection{Quantum evolution: wave function and energy levels}

In the following we obtain the solutions (wave functions) and the energy levels of the Schrödinger equation (98) in the EC noncommutative algebra. In the polar coordinate system $(r, \phi)$ with $x=r \cos \phi, y=r \sin \phi$, the angular momentum operator is represented by $L_{z}=-i \hbar \frac{\partial}{\partial \phi}$. Then the generalized stationary Schrödinger equation can be expressed as

$$
\begin{gathered}
{\left[-\frac{\hbar^{2}}{2 m^{*}}\left(\frac{\partial^{2}}{\partial r^{2}}+\frac{1}{r} \frac{\partial}{d r}+\frac{1}{r^{2}} \frac{\partial^{2}}{\partial \phi^{2}}\right)+i \hbar B_{h}(E) \frac{\partial}{\partial \phi}\right.} \\
\left.+\frac{K_{h}(E)}{2} r^{2}\right] \psi(r, \phi)=E \psi(r, \phi) .
\end{gathered}
$$

Due to the axial symmetry of Eq. (99), the wave function can be represented as

$\psi(r, \phi)=R(r) \mathrm{e}^{i m_{\phi} \phi}$,

where $m_{\phi}=0,1,2, \ldots$ By substituting Eq. (100) into Eq. (99), we obtain

$$
\begin{gathered}
r^{2} R^{\prime \prime}(r)+r R^{\prime}(r)+\left\{\frac{2 m^{*}}{\hbar^{2}}\left[E+m_{\phi} \hbar B_{h}(E)\right] r^{2}\right. \\
\left.-m_{\phi}^{2}-\frac{m^{*} K_{h}(E)}{\hbar^{2}} r^{4}\right\} R(r)=0,
\end{gathered}
$$

where $R^{\prime}(r)=\mathrm{d} R(r) / \mathrm{d} r$. By introducing a new radial coordinate $\xi$, defined as

$$
\xi=\frac{\left[m^{*} K_{h}(E)\right]^{1 / 4}}{\sqrt{\hbar}} r
$$

and by denoting

$$
C=\frac{2 \sqrt{m^{*}}}{\hbar} \frac{E+m_{\phi} \hbar B_{h}(E)}{\sqrt{K_{h}(E)}},
$$

Eq. (101) takes the form

$\xi^{2} \frac{\mathrm{d}^{2} R(\xi)}{\mathrm{d} \xi^{2}}+\xi \frac{\mathrm{d} R(\xi)}{\mathrm{d} \xi}+\left(C \xi^{2}-\xi^{4}-m_{\phi}^{2}\right) R(\xi)=0$,

or, equivalently,

$\xi \frac{\mathrm{d}}{\mathrm{d} \xi}\left(\xi \frac{\mathrm{d} R(\xi)}{\mathrm{d} \xi}\right)+\left(C \xi^{2}-\xi^{4}-m_{\phi}^{2}\right) R(\xi)=0$.

In the range of values of $\xi$ so that $C \xi^{2}>>\xi^{4}$, or, equivalently,

$\xi<<\frac{\left(4 m^{*}\right)^{1 / 4}\left[E+m_{\phi} \hbar B_{h}(E)\right]^{1 / 2}}{\hbar^{1 / 2} K_{h}^{1 / 4}(E)}$,

Eq. (104) becomes

$\xi^{2} \frac{\mathrm{d}^{2} R(\xi)}{\mathrm{d} \xi^{2}}+\xi \frac{\mathrm{d} R(\xi)}{\mathrm{d} \xi}+\left(C \xi^{2}-m_{\phi}^{2}\right) R(\xi)=0$,

and it has the general solution given by

$R_{m_{\phi}}(\xi)=c_{1} J_{m_{\phi}}(\sqrt{C} \xi)+c_{2} Y_{m_{\phi}}(\sqrt{C} \xi)$,

where $J_{m_{\phi}}(\xi)$ is the Bessel function of the first kind, while $Y_{m_{\phi}}(\xi)$ denotes the Bessel function of the second kind [68]. $c_{1}$ and $c_{2}$ denote two arbitrary integration constants. Since the function $Y_{m_{\phi}}(\xi)$ is singular at the origin, we must take $c_{2}=0$ in the solution (108). Therefore the general solution of Eq. (107) can be written as

$$
\begin{aligned}
R_{m_{\phi}}(\xi) & =c_{1} J_{m_{\phi}}(\sqrt{C} \xi) \\
& =c_{1} \sum_{l=0}^{\infty} \frac{(-1)^{l}}{l ! \Gamma\left(l+m_{\phi}+1\right)}\left(\frac{\sqrt{C} \xi}{2}\right)^{2 l+m_{\phi}} \\
& =\frac{c_{1}}{\pi} \int_{0}^{\pi} \cos \left(m_{\phi} \tau-\sqrt{C} \xi \sin \tau\right) d \tau,
\end{aligned}
$$

where $\Gamma(z)$ is the gamma function. For small values of the argument, the wave function behaves like $R_{m_{\phi}}(\xi) \approx$ 
$\left[1 / \Gamma\left(m_{\phi}+1\right)\right](\sqrt{C} \xi / 2)^{m_{\phi}}$, while for large values of the argument we have $R_{m_{\phi}}(\xi) \approx c_{1} \sqrt{2 / \pi \sqrt{C} \xi} \cos$ $\left(\sqrt{C} \xi-m_{\phi} \pi / 2-\pi / 4\right)+\mathrm{e}^{|\operatorname{Im}(\xi)|} O(1 /|\xi|)$, a relation valid for $|\arg \sqrt{C} \xi|<\pi$ [68].

In the interval $\left[0, \xi_{s}\right]$, the Bessel functions satisfy the condition $\int_{0}^{\xi_{s}} J_{m_{\phi}}\left(j_{m_{\phi} l} \frac{x}{\xi_{s}}\right) J_{m_{\phi}}\left(j_{m_{\phi}} n \frac{x}{\xi_{s}}\right) x \mathrm{~d} x=(1 / 2) \xi_{s}^{2}$ $\left[J_{m_{\phi}+1}\left(j_{m_{\phi} l}\right)\right]^{2} \delta_{l n}$, where $j_{m_{\phi} l}$ is the $l$ th zero of $J_{m_{\phi}}(\xi)$ [68]. There is a large literature on the zeros of the Bessel functions; for a review and some recent results see [69].

Now we consider the case in which in Eq. (105) the term $\xi^{4}$ cannot be neglected. To solve Eq. (105) we introduce a new coordinate $\zeta$ defined as $\zeta=\xi^{2}$. Then we obtain immediately $\xi \frac{\mathrm{d}}{\mathrm{d} \xi}=2 \zeta \frac{\mathrm{d}}{\mathrm{d} \zeta}, \xi \frac{\mathrm{d}}{\mathrm{d} \xi}\left(\xi \frac{\mathrm{d} R}{\mathrm{~d} \xi}\right)=4\left(\zeta \frac{\mathrm{d}^{2} R}{\mathrm{~d} \zeta^{2}}+\zeta \frac{\mathrm{d} R}{\mathrm{~d} \zeta}\right)$, and Eq. (105) takes the form

$\frac{\mathrm{d}^{2} R(\zeta)}{\mathrm{d} \zeta^{2}}+\frac{1}{\zeta} \frac{\mathrm{d} R(\zeta)}{\mathrm{d} \zeta}+\left(\frac{C-\zeta}{4 \zeta}-\frac{m_{\phi}^{2}}{4 \zeta^{2}}\right)=0$.

Next we introduce a new function $L\left(\zeta, m_{\phi}\right)$ by means of the transformation

$R(\zeta)=\zeta^{m_{\phi} / 2} \mathrm{e}^{-\zeta / 2} L\left(\zeta, m_{\phi}\right)$

Hence Eq. (110) becomes

$$
\begin{gathered}
\zeta \frac{\mathrm{d}^{2} L\left(\zeta, m_{\phi}\right)}{\mathrm{d} \zeta^{2}}+\left(m_{\phi}+1-\zeta\right) \frac{\mathrm{d} L\left(\zeta, m_{\phi}\right)}{d \zeta} \\
+\frac{1}{2}\left(\frac{C}{2}-m_{\phi}-1\right) L\left(\zeta, m_{\phi}\right)=0 .
\end{gathered}
$$

For

$n=\frac{1}{2}\left(\frac{C}{2}-m_{\phi}-1\right) \geq 0, \quad n \in \mathrm{N}$,

that is, for $n$ taking non-negative integer values, the wellbehaved solution of Eq. (112) is given by

$L\left(\zeta, m_{\phi}\right)=c L_{n}^{\left(m_{\phi}\right)}(\zeta)$

where $c$ is an arbitrary integration constant, and $L_{n}^{\left(m_{\phi}\right)}(\zeta)$ are the generalized Laguerre polynomials, defined as [68]

$L_{n}^{\left(m_{\phi}\right)}(\zeta)=\frac{\zeta^{m_{\phi}} \mathrm{e}^{\zeta}}{n !} \frac{\mathrm{d}^{n}}{\mathrm{~d} \zeta^{n}}\left(\mathrm{e}^{-\zeta} \zeta^{n+m_{\phi}}\right)$,

or, alternatively, as $L_{n}^{\left(m_{\phi}\right)}=x^{-m_{\phi}}\left(\frac{\mathrm{d}}{\mathrm{d} x}-1\right)^{n} \zeta^{n+m_{\phi}} / n$ ! Hence the physical solution of Eq. (104) can be obtained:

$R_{n}^{\left(m_{\phi}\right)}(\xi)=c \mathrm{e}^{-\xi^{2} / 2} \xi^{m_{\phi}} L_{n}^{\left(m_{\phi}\right)}\left(\xi^{2}\right)$.
The radial wave function must satisfy the normalization condition

$\int_{0}^{\infty}\left[R_{n}^{\left(m_{\phi}\right)}(r)\right]^{2} r \mathrm{~d} r=1$,

or, equivalently,

$c \frac{\hbar}{\left[m^{*} K_{h}(E)\right]^{1 / 2}} \int_{0}^{\infty} \xi^{2 m_{\phi}} \mathrm{e}^{-\xi^{2}}\left[L_{n}^{\left(m_{\phi}\right)}\left(\xi^{2}\right)\right]^{2} \xi \mathrm{d} \xi=1$.

By introducing a new variable $\xi^{2}=x, 2 \xi \mathrm{d} \xi=\mathrm{d} x$, we obtain

$\frac{c}{2} \frac{\hbar}{\left[m^{*} K_{h}(E)\right]^{1 / 2}} \int_{0}^{\infty} x^{m_{\phi}} \mathrm{e}^{-x}\left[L_{n}^{\left(m_{\phi}\right)}(x)\right]^{2} \mathrm{~d} x=1$.

By taking into account the mathematical identity $[2,68]$

$\int_{0}^{\infty} \mathrm{e}^{-x} x^{a}\left(L_{n}^{(a)}(x)\right)^{2} \mathrm{~d} x=(n+a) ! / n !$,

we find for the integration constant $c$ the expression

$c=\frac{2 n !}{\left(n+m_{\phi}\right) !} \frac{\left[m^{*} K_{h}(E)\right]^{1 / 2}}{\hbar}$.

The quantized energy levels of the free particle, and of the harmonic oscillator, can be obtained in the noncommutative energy dependent quantum mechanics as solutions of the algebraic equation

$\frac{\hbar}{\sqrt{m^{*}}}\left(2 n+m_{\phi}+1\right)=\frac{E+m_{\phi} \hbar B_{h}(E)}{\sqrt{K_{h}(E)}}$.

The commutative quantum mechanical limit for the harmonic oscillator is recovered, as one can see easily from Eqs. (56a)-(56c), when $E<<E_{0}$, giving $B_{h}(E)=0$ and $K_{h}(E)=k$, respectively. Then from Eq. (122) we immediately obtain

$E_{\mathrm{com}}=E_{n}^{\left(m_{\phi}\right)}=\hbar \omega\left(2 n+m_{\phi}+1\right)$,

where $\omega^{2}=k / m$. This relation gives the energy spectrum of the harmonic oscillator in commutative quantum mechanics [70].

6.3 The case of the free particle

Let us consider first the simple case of the free particle, with $B_{e}(E)=\left.B_{h}(E)\right|_{k=0}=B_{0}\left(E / E_{0}\right)^{\alpha}, k_{e}(E)=$ $\left.K_{h}(E)\right|_{k=0}=\left(k_{0} / 2\right)\left(E / E_{0}\right)^{2 \alpha}$, and $m^{*}=m$, respectively. 
Then from Eq. (122) it follows that the energy levels of the free particle are given by

$$
\begin{aligned}
E_{n}^{\left(m_{\phi}\right)}= & \left(\sqrt{\frac{2 m}{\hbar^{2} k_{0}}} E_{0}\right)^{1 /(\alpha-1)} \\
& \times \frac{E_{0}}{\left[2 n+\left(1-B_{0} \sqrt{2 m / k_{0}}\right) m_{\phi}+1\right]^{1 /(\alpha-1)}}, \alpha \neq 1 .
\end{aligned}
$$

For $\alpha=1$ the energy spectrum of the particle is continuous, and the two quantum numbers $n$ and $m_{\phi}$ must satisfy the condition $E_{0}=\hbar \sqrt{k_{0} / 2 m}\left[2 n+\left(1-B_{0} \sqrt{2 m / k_{0}}\right) m_{\phi}+1\right]$.

The wave function of the two-dimensional free particle in energy dependent noncommutative quantum mechanics is given by

$$
\begin{aligned}
R_{n}^{\left(m_{\phi}\right)}(r)= & c\left(\frac{m k_{0}}{2 \hbar^{2}}\right)^{\frac{m_{\phi}}{4}}\left(\frac{E}{E_{0}}\right)^{\frac{m_{\phi} \alpha}{2}} \mathrm{e}^{-\sqrt{\frac{m k_{0}}{8 \hbar^{2}}}\left(\frac{E}{E_{0}}\right)^{\alpha} r^{2}} \\
& \times r^{m_{\phi}} L_{n}^{\left(m_{\phi}\right)}\left[\sqrt{\frac{m k_{0}}{2 \hbar^{2}}}\left(\frac{E}{E_{0}}\right)^{\alpha} r^{2}\right] .
\end{aligned}
$$

In commutative quantum mechanics the wave function of a freely moving quantum particle with momentum $\vec{p}$ is given by $\Psi_{\vec{p}}=$ const. $\mathrm{e}^{i \vec{p} \cdot \vec{r} / \hbar}$. If we introduce the wave vector $\vec{K}$, defined by $\vec{K}=\vec{p} / \hbar$, then the wave function of the free particle is given by $\Psi_{\vec{K}}=$ const. $\mathrm{e}^{i \vec{K} \cdot \vec{r}}$. The energy spectrum of the particle is continuous, with $E=\vec{p}^{2} / 2 m=\hbar^{2} K^{2} / 2 m$. The evolution of the free particle in the energy-dependent noncommutative quantum mechanics is qualitatively different from the standard quantum mechanical case. The particle is not anymore "free", but its dynamics is determined by the presence of the effective potential generated by the noncommutative effects. Moreover, the energy levels are quantized in terms of two quantum numbers $n$ and $m_{\phi}$.

The ground state of the free particle corresponds to the choice $n=m_{\phi}=0$. Then the ground-state energy is given by

$E_{0}^{(0)}=\left(\frac{2 m}{\hbar^{2} k_{0}}\right)^{\frac{1}{2(\alpha-1)}} E_{0}^{\frac{\alpha}{\alpha-1}}, \alpha \neq 1$.

The radial wave function of the ground state of the free particle in energy-dependent quantum mechanics takes the form

$$
R_{0}^{(0)}(r)=c \mathrm{e}^{-\sqrt{\frac{m k_{0}}{8 \hbar^{2}}}\left(\frac{E}{E_{0}}\right)^{\alpha} r^{2}} .
$$

A possibility to test the energy-dependent noncommutative quantum mechanics would be through the study of the collision and scattering processes. Collisions are characterized by the differential cross section $\mathrm{d} \sigma / \mathrm{d} \Omega$, defined as the ratio of the number $N$ of particles scattered into direction $(\theta, \phi)$ per unit time per unit solid angle, divided by incident flux $j, \mathrm{~d} \sigma / \mathrm{d} \Omega=N / j[2,3]$. Usually one considers that the incident wave on the target corresponds to a free particle, and the scattering wave function is given by $\psi(\vec{r}) \sim \mathrm{e}^{i \vec{K} \cdot \vec{r}}+f(\theta) \mathrm{e}^{i \vec{K} \cdot \vec{r}} / r$. However, in energydependent quantum mechanics the wave function of the free particle at infinity cannot be described anymore as a simple plane wave. Hence, at least in principle, energy-dependent noncommutative effects could be determined and studied experimentally through their effects on the scattering cross sections in very high-energy particle collisions. A cross section dependent on the particle energies may be an indicator of the noncommutative quantum mechanical effects, and may provide an experimental method to detect the presence of the quantum spacetime.

\subsection{The harmonic oscillator}

Next we consider the harmonic oscillator problem in the energy coupling model of the energy-dependent noncommutative quantum mechanics. By taking into account the explicit forms of the effective mass, effective magnetic field and effective elastic constant as given by Eqs. (56a)-(56c), it follows that the energy spectrum of the harmonic oscillator is obtained as a solution of the nonlinear algebraic equation given by

$$
\begin{gathered}
\hbar \omega \sqrt{1+\frac{m^{2} \omega^{2} \theta_{0}}{4 \hbar^{2}} x^{\beta}\left(2 n+m_{\phi}+1\right)} \\
=\frac{E_{0} x+\frac{m_{\phi} \omega^{2}}{2}\left[\frac{\eta_{0}}{k} x^{\alpha}+m \theta_{0} x^{\beta}\right]}{\sqrt{1+\frac{\eta_{0}^{2}}{8 m^{2} \omega^{2} \hbar^{2}} x^{2 \alpha}}},
\end{gathered}
$$

where we have denoted $x=E / E_{0}$, and $\omega=\sqrt{k / m}$. In order to solve this equation we need to fix, from physical considerations, the numerical values of the quantities $\alpha$ and $\beta$. For arbitrary values of $\alpha$ and $\beta$ the energy levels can be obtained generally only by using numerical methods. In the first order approximation we obtain

$$
\begin{aligned}
& \hbar \omega\left(1+\frac{m^{2} \omega^{2} \theta_{0}}{8 \hbar^{2}} x^{\beta}\right)\left(2 n+m_{\phi}+1\right) \\
& =E_{0} x+\frac{m_{\phi} \omega^{2}}{2}\left[\frac{\eta_{0}}{k} x^{\alpha}+m \theta_{0} x^{\beta}\right]-\frac{E_{0} \eta_{0}^{2}}{16 m^{2} \omega^{2} \hbar^{2}} x^{2 \alpha+1},
\end{aligned}
$$


or, equivalently,

$$
\begin{aligned}
\left(1+\frac{m^{2} \omega^{2} \theta_{0}}{8 \hbar^{2}} x^{\beta}\right) E_{\mathrm{com}}= & E_{0} x+\frac{m_{\phi} \omega^{2}}{2} \\
& \times\left[\frac{\eta_{0}}{k} x^{\alpha}+m \theta_{0} x^{\beta}\right] \\
& -\frac{E_{0} \eta_{0}^{2}}{16 m^{2} \omega^{2} \hbar^{2}} x^{2 \alpha+1}
\end{aligned}
$$

where $E_{\text {com }}$ denotes the energy levels of the harmonic oscillator in the commutative formulation of quantum mechanics. In the simple case $\alpha=\beta=1$, in the first approximation we obtain for the energy levels the algebraic equation

$$
\begin{aligned}
\left(1+\frac{m^{2} \omega^{2} \theta_{0}}{8 \hbar^{2}} x\right) E_{\mathrm{com}}= & {\left[E_{0}+\frac{m_{\phi} \omega^{2}}{2}\left(\frac{\eta_{0}}{k}+m \theta_{0}\right)\right] x } \\
& -\frac{E_{0} \eta_{0}^{2}}{16 m^{2} \omega^{2} \hbar^{2}} x^{3}
\end{aligned}
$$

By neglecting the term $x^{3}$, we obtain the energy levels in the energy-dependent noncommutative quantum mechanics in the first order approximation:

$$
E_{n}^{\left(m_{\phi}\right)} \approx \frac{E_{\mathrm{com}}}{E_{0}+\frac{m_{\phi} \omega^{2}}{2}\left(\frac{\eta_{0}}{k}+m \theta_{0}\right)-\frac{m^{2} \omega^{2} \theta_{0}}{8 \hbar^{2}} E_{\mathrm{com}}} .
$$

The wave function of the ground state of the energydependent harmonic oscillator in noncommutative quantum mechanics, corresponding to $n=m_{\phi}=0$, can be written as

$$
R_{0}^{(0)}(r) \sim \exp \left\{-\frac{m \omega}{\hbar}\left[\frac{1+\frac{\eta_{0}^{2}}{8 m^{2} \omega^{2} \hbar^{2}}\left(\frac{E}{E_{0}}\right)^{2 \alpha}}{1+\frac{m^{2} \omega^{2} \theta_{0}}{4 \hbar^{2}}\left(\frac{E}{E_{0}}\right)^{\beta}}\right]^{1 / 2} \frac{r^{2}}{2}\right\}
$$

In the limit $E<<E_{0}$, we recover the standard commutative result for the eave function of the ground state of the quantum mechanical harmonic oscillator, $\psi(r) \sim$ $\exp \left\{-\frac{m \omega}{\hbar} \frac{r^{2}}{2}\right\}[2,3]$.

The wave function of the ground state of the harmonic oscillator in the energy-dependent noncommutative quantum mechanics can be written in a form analogous to the commutative case by introducing the effective energy-depending frequency $\omega_{\text {eff }}$, defined as

$\omega_{\mathrm{eff}}(E)=\omega\left[\frac{1+\frac{\eta_{0}^{2}}{8 m^{2} \omega^{2} \hbar^{2}}\left(\frac{E}{E_{0}}\right)^{2 \alpha}}{1+\frac{m^{2} \omega^{2} \theta_{0}}{4 \hbar^{2}}\left(\frac{E}{E_{0}}\right)^{\beta}}\right]^{1 / 2}$.
Then the wave function of the ground state of the harmonic oscillator can be written as

$R_{0}^{(0)}(r) \sim \exp \left[-\frac{m \omega_{\mathrm{eff}}(E)}{\hbar} \frac{r^{2}}{2}\right]$

Hence we have completely solved the problem of the quantum mechanical motion of the free particle, and of a particle in a harmonic potential, in the noncommutative quantum mechanics with energy-dependent strengths.

\section{Quantum evolution in the energy operator (EO) energy-dependent noncommutative geometry}

Finally, we will consider in detail the third possibility of constructing quantum mechanics in the framework of energydependent noncommutative geometry. This approach consists in mapping the energy in the noncommutative algebra to an operator. As we have already discussed, we have two possibilities to develop such an approach, by mapping the energy to the time operator, or to the particle Hamiltonian. Under the assumption of a power-law dependence on energy of the noncommutative strengths, in the general case these maps lead to a fractional Schrödinger equation. In the following we will first write down the basic fractional Schrö dinger equations for the free particle and the harmonic oscillator case, and after that we will proceed to a detailed study of their properties. We will concentrate on the models obtained by the time operator representation of the energy. But before proceeding to discuss the physical implications of the generalized Schrödinger equation with fractional operators, we will present o very brief summary of the basic properties of the fractional calculus.

\subsection{Fractional calculus: a brief review}

For $\alpha, \beta \notin N$, the quantum mechanical model introduced in the previous sections, based on the power-law energy dependence of the noncommutative strengths, leads to the interesting question of the mathematical and physical interpretation of the operators of the form $\left[\left(i h / E_{0}\right) \partial / \partial t\right]^{\alpha}$. It turns out that such operators can be written is terms of a fractional derivative as

$\eta\left(i \hbar \frac{\partial}{\partial t}\right) \Psi=\eta_{0}\left(\frac{i \hbar}{E_{0}}\right)^{\alpha} \frac{\partial^{\alpha}}{\partial t^{\alpha}}=\eta_{0}\left(\frac{i \hbar}{E_{0}}\right)^{\alpha} D_{t}^{\alpha} \Psi$

where $D_{t}^{\alpha}=\partial^{\alpha} / \partial t^{\alpha}$ is the fractional derivative of $\Psi$ of the order $\alpha>0$, which can be defined in terms of the fractional integral $D_{t}^{-m} \Psi(t)$ as [71-73]

$D_{t}^{\alpha} \Psi(t)=D_{t}^{m}\left[D_{t}^{-(m-\alpha)} \Psi(t)\right]$ 
Hence in fractional calculus a fractional derivative is defined via a fractional integral. By interpreting the time derivative operators as fractional derivatives we obtain the fractional Schrödinger equations as given by the equations presented in the next section.

There are several definitions of the fractional derivative that have been intensively investigated in the mathematical and physical literature. For example, the Caputo fractional derivative is defined as

${ }_{\alpha}^{C} D_{t}^{\alpha} \Psi(t)=\frac{1}{\Gamma(n-\alpha)} \int_{a}^{t} \frac{\Psi^{(n)}(\tau) \mathrm{d} \tau}{(t-\tau)^{\alpha+1-n}}$.

Given a function $f(x)=\sum_{k=0}^{\infty} a_{k} x^{k \alpha}$, its fractional Caputo derivative can be obtained according to

$\frac{\mathrm{d}^{\alpha}}{\mathrm{d} x^{\alpha}} f(x)=\sum_{k=0}^{\infty} a_{k+1} \frac{\Gamma[1+(k+1) \alpha]}{\Gamma(1+k \alpha)} x^{k \alpha}$,

where $\Gamma(z)$ is the gamma function defined as $\Gamma(z)=$ $\int_{0}^{\infty} t^{z-1} \mathrm{e}^{-t} \mathrm{~d} t$, with the property $\Gamma(1+z)=z \Gamma(z)[73]$.

Consequently, we obtain the definition of the Caputo fractional derivative of the exponential function:

$$
\begin{aligned}
\frac{\mathrm{d}^{\alpha}}{\mathrm{d} x^{\alpha}} \mathrm{e}^{x} & =\frac{\mathrm{d}^{\alpha}}{\mathrm{d} x^{\alpha}} \sum_{n=0}^{\infty} \frac{x^{n}}{n !}=\sum_{n=1}^{\infty} \frac{x^{n-\alpha}}{\Gamma(1+n-\alpha)} \\
& =x^{1-\alpha} E_{1,2-\alpha}(x),
\end{aligned}
$$

where $E_{1,2-\alpha}(x)$ is the generalized Mittag-Leffler function, defined as $E_{\alpha, \beta}(z)=\sum_{n=0}^{\infty} z^{n} / \Gamma(n \alpha+\beta)$ [73].

Another definition of the fractional derivative is the Liouville definition, which implies [73]

$$
\frac{\mathrm{d}^{\alpha}}{\mathrm{d} x^{\alpha}} \mathrm{e}^{k x}=k^{\alpha} \mathrm{e}^{k x}, \quad k \geq 0
$$

Finally, we also point out the definition of the left-sided Riemann-Liouville fractional integral of order $v$ of the function $f(t)$, which is defined as

${ }_{a} D^{\nu} f(x):=\frac{1}{\Gamma(n-v)} \frac{\mathrm{d}^{n}}{\mathrm{~d} x^{n}}\left(\int_{0}^{x} \frac{f(\tau}{x-\tau}^{\nu+1-n} \mathrm{~d} \tau\right)$,

a definition which is valid for $n-1<v<n \in \mathbb{N}$ [73].

\subsection{The fractional Schrödinger equation}

In the present section we will consider the evolution of a system in the energy operator representation of the energydependent noncommutative quantum mechanics. We will restrict again our analysis to the two-dimensional case only. The generalized fractional two-dimensional Schrödinger equation can then be expressed as $i \hbar \frac{\partial}{\partial t} \Psi(x, y, t)=\widehat{H} \Psi(x, y, t)$,

where

$\widehat{H}=-\frac{\hbar^{2}}{2 m_{\ell}^{*}} \Delta_{2}+V_{\mathrm{eff}}$,

with

$V_{\mathrm{eff}}=V(x, y)-B_{\ell} D_{\ell}^{\alpha} L_{z}+\frac{1}{2} K_{\ell} D_{\ell}^{2 \alpha}\left(x^{2}+y^{2}\right)$

and we have denoted

$$
\begin{aligned}
\frac{1}{m_{\ell}^{*}} & =\frac{1}{m}+\frac{2 \kappa_{\ell}}{\hbar^{2}}, \quad \kappa_{\ell}=\frac{k \theta_{\alpha}^{2}}{8}, \\
B_{\ell} & =B_{\alpha}^{\ell}+\frac{k \theta_{\alpha}}{2 \hbar}, \quad K_{\ell}=k+k_{\ell}^{\alpha},
\end{aligned}
$$

where $\ell=I$, II denote the two different operator representations of the EO noncommutative algebra. $D_{I}^{\alpha} \equiv\left(\frac{\partial}{\partial t}\right)^{\alpha}$ and $D_{I I}^{\alpha} \equiv D_{x}^{2 \alpha}+D_{y}^{2 \alpha}$ are the fractional derivatives with respect to time and space when $\alpha$ is a rational integer.

In the following we will concentrate only on the time operator representation of the noncommutative quantum mechanics with energy-dependent strengths, namely $\ell=I$. Thus, by representing the wave function as

$\Psi(t, x, y)=\mathrm{e}^{-\frac{i}{\hbar} E t} \psi(x, y)$

the Schrödinger equation becomes a fractional differential equation given by

$$
\begin{aligned}
& {\left[-\frac{\hbar^{2}}{2 m_{\ell}^{*}} \triangle_{2}+V(x, y)-B_{\ell} \mathcal{D}_{\alpha}(t) L_{z}\right.} \\
& \left.\quad+\frac{1}{2} K_{\ell} \mathcal{D}_{2 \alpha}(t)\left(x^{2}+y^{2}\right)\right] \psi(x, y)=E \psi(x, y),
\end{aligned}
$$

where

$\mathcal{D}_{\alpha}(t)=\mathrm{e}^{\frac{i}{\hbar} E t} D_{t}^{\alpha} \mathrm{e}^{-\frac{i}{\hbar} E t}, \quad \mathcal{D}_{2 \alpha}(t)=\mathrm{e}^{\frac{i}{\hbar} E t} D_{t}^{2 \alpha} \mathrm{e}^{-\frac{i}{\hbar} E t}$.

If the function $\mathrm{e}^{-\frac{i}{\hbar} E t}$ is an eigenfunction of the fractional derivation operators $D^{\alpha}$ and $D^{2 \alpha}$, so that

$D_{t}^{\alpha} \mathrm{e}^{-\frac{i}{\hbar} E t}=a_{\alpha} \mathrm{e}^{-\frac{i}{\hbar} E t}, \quad D_{t}^{2 \alpha} \mathrm{e}^{-\frac{i}{\hbar} E t}=a_{2 \alpha} \mathrm{e}^{-\frac{i}{\hbar} E t}$,

where $a_{\alpha}$ and $a_{2 \alpha}$ are constants, the separation of the time variable can be performed in the noncommutative fractional Schrö dinger equation with energy-dependent noncommutative strengths. 


\subsection{The free particle: the case $\alpha=1$}

For simplicity, in the following we investigate the quantum dynamics in the energy operator representation only in the case of the free particle, by assuming $V(\widehat{x}, \widehat{y})=0$. Therefore $k=0$, and the effective mass of the particle coincides with the ordinary mass, $m_{l}^{*}=m$. Moreover, for simplicity we will restrict our analysis to the choice $\alpha=1$. Then $\mathcal{D}_{1}(t)=-\frac{i}{\hbar} E$ and $\mathcal{D}_{2}(t)=-\frac{1}{\hbar^{2}} E^{2}$, respectively.

Explicitly, the Schrödinger equation describing the motion of the free particle in the energy-dependent noncommutative geometry takes the form

$$
\begin{aligned}
& i \hbar \frac{\partial}{\partial t} \Psi(t, x, y)-\frac{i \eta_{0}}{2 m E_{0}} \hat{L}_{z} \frac{\partial}{\partial t} \Psi(t, x, y) \\
& \quad+\frac{\eta_{0}^{2}}{8 m E_{0}^{2}}\left(x^{2}+y^{2}\right) \frac{\partial^{2}}{\partial t^{2}} \Psi(t, x, y)=-\frac{\hbar^{2}}{2 m} \Delta_{2} \Psi(t, x, y) .
\end{aligned}
$$

In the polar coordinate system $(r, \phi)$ with $r=r \cos \phi$, $y=r \sin \phi$, we have

$\hat{L}_{z}=\frac{\hbar}{i} \frac{\partial}{\partial \phi}$,

and

$\Delta_{2}=\frac{1}{r} \frac{\partial}{\partial r}\left(r \frac{\partial}{d r}\right)+\frac{1}{r^{2}} \frac{\partial^{2}}{\partial \phi^{2}}$,

respectively.

By introducing for the wave function the representation $\Psi(t, x, y)=\mathrm{e}^{-(i / \hbar) E t} \psi(r, \phi)$, and, similarly to the previous section, representing the reduced wave function as $\psi(r, \phi)=$ $R(r) \mathrm{e}^{i m_{\phi} \phi}, m_{\phi} \in \mathbb{N}$, it follows that Eq. (148) takes the form

$$
\begin{gathered}
r^{2} R^{\prime \prime}(r)+r R^{\prime}(r)+\left[\frac{2 m}{\hbar^{2}} E\left(1+m_{\phi} B_{I}\right) r^{2}\right. \\
\left.-\frac{m K_{I}}{\hbar^{4}} E^{2} r^{4}-m_{\phi}^{2}\right] R(r)=0 .
\end{gathered}
$$

By introducing a new independent variable $\xi$, defined as

$$
r=\frac{\hbar}{\left(m K_{I} E^{2}\right)^{1 / 4}} \xi
$$

and by denoting

$\sigma=\frac{2 \sqrt{m}\left(1+m_{\phi} B_{I}\right)}{\sqrt{K_{I}}}$,

Eq. (154) takes the form

$\xi^{2} \frac{\mathrm{d}^{2} R}{\mathrm{~d} \xi^{2}}+\xi \frac{\mathrm{d} R}{\mathrm{~d} \xi}+\left(\sigma \xi^{2}-\xi^{4}-m_{\phi}^{2}\right) R=0$,
When $\sigma \xi^{2}>>\xi^{4}$ or, equivalently, $\xi<<(4 m)^{1 / 4}$ $\left(1+m_{\phi} B_{I}\right)^{1 / 2} /\left(K_{I}\right)^{1 / 4}$, that is, when the noncommutative effects can be neglected, in the standard quantum mechanical limit we obtain the solution of Eq. (157):

$R(\xi)=c_{1} J_{n}(\xi)+c_{2} Y_{n}(\xi)$,

where $c_{1}$ and $c_{2}$ are arbitrary integration constants, and $J_{n}(\xi)$ and $Y_{n}(\xi)$ are the Bessel function of the first kind and the Bessel function of the second kind [68], respectively. We have already discussed in detail the behavior of the wave function in this case in the previous section.

If the term $\xi^{4}$ cannot be neglected as compared to $\sigma \xi^{2}$, then the general solution of Eq. (157) is given by

$R_{n}^{\left(m_{\phi}\right)}(\xi)=c \mathrm{e}^{-\xi^{2} / 2} \xi^{m_{\phi}} L_{n}^{\left(m_{\phi}\right)}\left(\xi^{2}\right)$,

that is, the same form of the solution as the one already considered when discussing the evolution of the free particle and of the harmonic oscillator in the energy coupling model of the energy-dependent noncommutative quantum mechanics. The wave function must be finite at the origin. The normalization and other properties of the wave function are similar to the ones already investigated in the previous section.

\section{Discussions and final remarks}

In the present paper we have considered the quantum mechanical implications of a noncommutative geometric model in which the strengths of the noncommutative parameters are energy dependent. From a physical point of view such an approach may be justified since the effects of the noncommutativity of the spacetime are expected to become apparent at extremely high energies, of the order of the Planck energy, and at distance scales of the order of the Planck length. By assuming an energy-dependent noncommutativity we obtain a smooth transition between the maximally noncommutative geometry at the Planck scale, and its commutative ordinary quantum mechanical version, which can be interpreted as the low-energy limit of the noncommutative high-energy quantum mechanics. Hence this approach unifies in a single formalism two apparently distinct approaches, the noncommutative and commutative versions of quantum mechanics, respectively, and generally leads to an energy-dependent Schrödinger equation, as already considered in the literature [61-67].

One of the important question related to the formalism developed in the present paper is related to the physical implications of the obtained results. In the standard approach to noncommutative geometry, by using the linearity of the $D$ map, one could find a representation of the noncommutative observables as operators acting on the conventional Hilbert 
space of ordinary quantum mechanics. More exactly, the $D$ map converts the noncommutative system into a modified commutative quantum mechanical system that contains an explicit dependence of the Hamiltonian on the noncommutative parameters, and on the particular $D$ map used to obtain the representation. The states of the considered quantum system are then wave functions in the ordinary Hilbert space; the dynamics is determined by the standard Schrödinger equation with a modified Hamiltonian that depends on the noncommutative strengths $\theta$ and $\eta$ [26]. Even though the mathematical formalism is dependent on the functional form of the adopted $D$ map that is used to realize the noncommutativecommutative conversion, this is not the case for physical predictions of the theory such as expectation values and probability distributions [26]. On the other hand it is important to point the fact that the standard formalism in which the energy dependence is ignored is not manifestly invariant under a modification of the $D$ map.

In the energy-dependent approach to noncommutative geometry after performing the $D$ map we arrive at an energy-dependent Schrödinger equation, which contains some energy-dependent potentials $V(x, E, \theta, \eta)$. In order to obtain a consistent physical interpretation we need to redefine the probability density, the normalization condition and the expectation values of the physical observables. For example, in order to be sure that a solution of the Schrödinger equation associated with a stationary energy $E$ is normalizable the following two conditions must hold simultaneously [62]:

$1-\frac{\partial V(x, E, \theta, \eta)}{\partial E} \geq 0, x \in D, N(\psi)<\infty$.

Moreover, in opposition to the standard case of an energyindependent potential, the nonnegativity and the existence of the norm integral must hold at the same time. The modified forms of the probability density and of the probability current also lead to adjustments in the scalar product and the norm that do not appear in standard quantum mechanics. Similarly to the case of standard noncommutative quantum mechanics we also expect that, like in the energy-independent case, our present formalism is not invariant under a change in the functional form of the $D$ map. The phase-space formulation of a noncommutative extension of quantum mechanics in arbitrary dimensions, with both spatial and momentum noncommutativities, was considered in [26]. By considering a covariant generalization of the Weyl-Wigner transform and of the Darboux $D$ map, an isomorphism between the operator and the phase-space representations of the extended Heisenberg algebra was constructed. This map allows one to develop a systematic approach to deriving the entire structure of noncommutative quantum mechanics in phase space. More importantly, it turns out that the entire formalism is independent of the particular choice of the Darboux map. The extension of the results of [26] to the energy-dependent case would help to clarify the mathematical structure and physical properties of energy-dependent noncommutative quantum mechanics.

In order to implement the idea of energy-dependent noncommutativity we need to specify the relevant energy scales. In the present work we have assumed a two scale and a single energy scale model. Moreover, we have limited our investigations to the case in which the noncommutative strengths have a simple power-law dependence on the energy. In the first approach the energy dependence of the noncommutative strengths is determined by a specific energy scale, which is related to the energy of the quantum fluctuations that modify the geometry. This approach may be valid to describe physics very nearby the Planck scale, where the vacuum energy may be the dominant physical effect influencing the quantum evolution of particles in the noncommutative geometric setting. In this context we have considered the dynamics of two simple but important quantum systems, the free particle, and the harmonic oscillator, respectively. The physical characteristics of the evolution are strongly dependent on the energy of the quantum fluctuations, with the oscillations frequencies effectively determined by the spacetime fluctuation scale.

In our second model we have assumed that all the noncommutative effects can be described by means of the particle energy scale, which is the unique scale determining the physical implications of noncommutative geometry. The choice of a single energy scale allows the smooth transition from the noncommutative algebra of the Planck length to the commutative Heisenberg algebra of the ordinary quantum mechanics that gives an excellent description of the physical processes on the length and energy scales of the atoms and molecules, and for the standard model of elementary particles. In this case the basic physical parameters of the of the quantum dynamics of the free particles and of the harmonic oscillator are energy dependent, with the oscillation frequencies described by complicated functions of the particle energy. Such an energy dependence of the basic physical parameters of the quantum processes may have a significant impact on the high-energy evolution of the quantum particles.

Perhaps the most interesting physical implications are obtained in the framework of our third approach, which consists in mapping the energy with a quantum operator. There are two such possibilities we have briefly discussed, namely, mapping the energy with the time derivative operator, and with the Hamiltonian of the free particle (its kinetic energy). The corresponding Schrödinger equation changes its mathematical form, content an interpretation, becoming a fractional differential equation, in which the ordinary derivatives of quantum mechanics are substituted by fractional ones.

Fractional Schrödinger equations have been introduced some time ago in the physical literature [74], and presently they are becoming a very active field of research in both 
physics and mathematics [74-93]. For an extensive review of fractional quantum mechanics see [94]. A typical example of a fractional Schrödinger equation is given by the equation [74]

$i \hbar \frac{\partial \psi(\vec{r}, t)}{\partial t}=D_{\alpha}\left(-\hbar^{2} \Delta\right)^{\alpha / 2} \psi(\vec{r}, t)+V(\vec{r}, t) \psi(\vec{r}, t)$,

where $D_{\alpha}$ is a constant, and $\alpha$ is an arbitrary number. The fractional Hamilton operator is Hermitic, and a parity conservation law for fractional quantum mechanics can also be established. The energy spectra of a hydrogenlike atom can also be obtained, while in this approach the fractional oscillator with the Hamiltonian

$H_{\alpha, \beta}=D_{\alpha}|p|^{\alpha}+q^{2}|x|^{\beta}$,

where $\alpha, \beta$, and $q$ are constants, has the energy levels quantized according to the rule [74]

$E_{n}=\left[\frac{\pi \hbar \beta D_{\alpha}^{1 / \alpha} q^{2 / \beta}}{2 B\left(\frac{1}{\beta}, \frac{1}{\alpha}+1\right)}\right]^{\frac{\alpha \beta}{\alpha+\beta}}\left(n+\frac{1}{2}\right)^{\frac{\alpha \beta}{\alpha+\beta}}$,

where the $B(a, b)$ function is defined by the integral representation $B(a, b)=\int_{0}^{1} \mathrm{~d} u u^{a-1}(1-u)^{b-1}$ [68], and $\alpha$ and $\beta$ are arbitrary numerical parameters. As for the physical origin of the fractional derivatives, in $[95,96]$ it was shown that it originates from the path integral approach to quantum mechanics. More exactly, the path integral over Brownian trajectories gives the standard Schr ödinger equation of quantum mechanics, while the path integral over Lévy trajectories generates the fractional Schrödinger equation. In the present paper we have outlined the possibility of another physical path towards the fractional Schrödinger equation, namely, the framework of the quantum operator approach to energy-dependent noncommutative geometry.

An interesting theoretical question in the field of noncommutative quantum mechanics is the problem of the nonlocality generated by the dynamical noncommutativity. This problem was investigated in [50] for a noncommutative quantum system with the coordinates satisfying the commutation relations $\left[\widehat{q}^{i}, \widehat{q}^{j}\right]=i \theta f(\sigma) \epsilon^{i j}$, where $f(\sigma)$ is a function of the physical parameter $\sigma$, which could represent, for example, position, spin, or energy. Then for the uncertainty relation between the coordinate operators $\widehat{x}$ and $\widehat{y}$ we obtain the uncertainty relation $(\Delta \widehat{x})_{\Psi}(\Delta \widehat{x})_{\Psi} \geq(\theta / 2)|\langle\Psi|f(\sigma)| \Psi\rangle|$ [50]. As was pointed out in [50], if $\mid \Psi>$ represents a stationary state of the quantum system, that is, an eigenstate of the Hamiltonian, it follows that the nonlocality induced by the noncommutativity of the coordinates will be a function of the energy. Moreover, in the present approach to noncommutative quantum mechanics, the noncommutative strength $\theta$ is an explicit function of the energy, and therefore an explicit dependence of the nonlocality on the energy always appears. As a particular case we consider $f(\sigma)=1$, that is, the noncommutative strengths depend only on the energy. Then, by taking into account the normalization of the wave function we obtain $(\Delta \widehat{x})_{\Psi}(\Delta \widehat{x})_{\Psi} \geq(\theta(E) / 2)$ or, by considering the explicit choice of the energy dependence of $\theta$ adopted in the present study, $(\Delta \widehat{x})_{\Psi}(\Delta \widehat{x})_{\Psi} \geq\left(\theta_{0} / 2\right)\left(E / E_{0}\right)^{\beta}$. Hence when $E<<E_{0},(\Delta \widehat{x})_{\Psi}(\Delta \widehat{x})_{\Psi} \approx 0$, and we recover the standard quantum mechanical result. In the case of the harmonic oscillator the uncertainty relations for the noncommutative coordinates can be obtained for $f(\sigma)=\sigma$ as $(\Delta \widehat{x})_{\Psi}(\Delta \widehat{x})_{\Psi} \geq O\left(\theta^{4}\right)$, that is, nonlocality does not appear in higher orders of $\theta$ [50]. For the case $f(\sigma)=\sigma^{2}$, one finds $(\Delta \widehat{x})_{\Psi}(\Delta \widehat{x})_{\Psi} \geq\left(\theta / 2 \omega_{\sigma}\right)(n+1 / 2)+O\left(\theta^{3}\right)$, where $\omega_{\eta}$ is the oscillation frequency of the $\sigma$-dependent potential term in the total Hamiltonian, given by $V(\sigma)=\omega_{\sigma}^{2} \sigma^{2} / 2$ [50].

A central question in the noncommutative extensions of quantum mechanics is the likelihood of its observational or experimental testing. A possibility of detecting the existence of the noncommutative phase space by using the AharonovBohm effect was suggested in [33]. As we have already seen the noncommutativity of the momenta leads to the generation of an effective magnetic field and of an effective flux. In a mesoscopic ring this flux induces a persistent current. By using this effect it may be possible to detect the effective magnetic flux generated by the presence of the noncommutative phase space, even if it is very weak. Persistent currents and magnetic fluxes in mesoscopic rings can be studied by using experimental methods developed in nanotechnology [33]. The dynamics of a free electron in the two-dimensional noncommutative phase space is equivalent to the evolution of the electron in an effective magnetic field, induced by the effects of the noncommutativity of the coordinates and momenta.

For the motion of a free electron in the noncommutative phase space, the Hamiltonian can be obtained:

$H_{n c}=\frac{1}{2 m}\left(\widehat{p}_{x}^{2}+\widehat{p}_{y}^{2}\right)=\frac{1}{2 m^{*}}\left[\left(p_{x}+e A_{x}\right)^{2}+\left(p_{y}+e A_{y}\right)^{2}\right]$,

where we have denoted by $m^{*}=m / \alpha$ the effective mass in the noncommutative phase space. The parameter $\alpha$ is defined through the relation $\theta \eta=2 \hbar^{2} \alpha^{2}\left(1-\alpha^{2}\right)$. The components $A_{x}$ and $A_{y}$ of the effective vector potential $\vec{A}$ are given by $A_{x}=\left(\eta / 2 e \alpha^{2} \hbar\right) y$ and $A_{y}=-\left(\eta / 2 e \alpha^{2} \hbar\right) x$, respectively [33], while the effective magnetic field is obtained in the form $B_{z}=\eta / e \alpha^{2} \hbar$.

A possibility of experimentally implementing a method that could detect noncommutative quantum mechanical 
effects consists in considering a one-dimensional ring in an external magnetic field $\vec{B}$, oriented along the axis of the ring. $\vec{B}$ is constant inside $r_{c}<R$ (ring radius), which implies that the electrons are located only in the field-free region of the small ring. Moreover, the quantum electronic states are functions of the total magnetic flux crossing the ring only. By introducing a polar coordinate system by means of the definitions $x=R \cos \varphi, y=R \sin \varphi$, we obtain for the Hamiltonian of the electrons the expression [33]

$$
H_{n c}=-\frac{\hbar^{2}}{2 m^{*} R^{2}}\left[\frac{\partial}{\partial \varphi}+i\left(\frac{\phi}{\phi_{0}}-\frac{\phi_{n c}}{\phi_{0}}\right)\right]^{2}-\frac{3 \hbar^{2}}{8 m^{*} R^{2}} \frac{\phi_{n c}^{2}}{\phi_{0}^{2}},
$$

where $\phi_{n c}=2 \pi R^{2} \eta / e \hbar \alpha^{2}$ represents an effective magnetic flux coming from the noncommutative phase space, while $\phi_{0}=h / e$ is the quantum of the magnetic flux. By $\phi$ we have denoted the external magnetic flux in the ring. Hence noncommutative effects generate a persistent current in the ring, which depends on the external and the effective magnetic fluxes, respectively. The relation between the persistent current and the magnetic flux may provide a method to detect the existence of noncommutative quantum mechanical effects. Hence by considering a mesoscopic ring system in the presence of an external magnetic field, and by studying the relation between the persistent current and the external magnetic flux $\varphi$ one can infer the possible existence of noncommutative quantum mechanical effects [33]. The theoretical model behind this experimental procedure can be easily reformulated by taking into account the variation of $\eta$ with the energy of the electrons. Hence the study of the persistent currents in mesoscopic systems by using experimental techniques already existent in nanotechnology may open the possibility of proving the existence of new quantum mechanical physical structures that becomes dominant at high particle energies.

The investigation of the spacetime structure and physical processes at very high energies and small microscopic length scales may open the possibility of a deeper understanding of the nature of the fundamental interactions and of their mathematical description. In the present work we have developed some basic tools that could help to give some new insights into the complex problem of the nature of the quantum dynamical evolution processes at different energy scales, and of their physical implications.

Acknowledgements We would like to thank the two anonymous reviewers for their comments and suggestions, which helped us to significantly improve our manuscript. T. H. would like to thank the Yat Sen School of the Sun Yat Sen University in Guangzhou, P. R. China, for the kind hospitality offered during the preparation of this work. S.-D. L. thanks the Natural Science Foundation of Guangdong Province for financial support (grant No. 2016A030313313).
Data Availability Statement This manuscript has no associated data or the data will not be deposited. [Authors' comment: Since this is a theoretical study no data are available.]

Open Access This article is distributed under the terms of the Creative Commons Attribution 4.0 International License (http://creativecomm ons.org/licenses/by/4.0/), which permits unrestricted use, distribution, and reproduction in any medium, provided you give appropriate credit to the original author(s) and the source, provide a link to the Creative Commons license, and indicate if changes were made.

Funded by $\mathrm{SCOAP}^{3}$.

\section{References}

1. S. Doplicher, K. Fredenhagen, J.E. Roberts, Commun. Math. Phys. 172, 187 (1995)

2. A. Messiah, Quantum Mechanics (Dover Publications, New York, 1999)

3. L.D. Landau, E.M. Lifshitz, Quantum Mechanics: Non-relativistic Theory (Butterworth-Heinemann, Oxford, 2003)

4. S.-D. Liang, Quantum Tunneling and Field Emission Theories (World Scientific, London, 2014)

5. K. Fredenhagen, Rev. Math. Phys. 7, 559 (1995)

6. H.S. Snyder, Phys. Rev. 71, 38 (1947)

7. C.N. Yang, Phys. Rev. 72, 874 (1947)

8. A. Connes, Inst. Hautes Études Sci. Publ. Math. 62, 257 (1985)

9. V. G. Drinfel'd, Proc. of the International Congress of Mathematicians (Berkeley, 1986). American Mathematical Society (1987)

10. S.L. Woronowicz, Publ. Res. Inst. Math. Sci. 23, 117 (1987)

11. S.L. Woronowicz, Commun. Math. Phys. 111, 613 (1987)

12. N. Seiberg, E. Witten, JHEP 9909, 032 (1999)

13. O. Bertolami, L. Guisado, JHEP 0312, 013 (2003)

14. M.R. Douglas, N.A. Nekrasov, Rev. Mod. Phys. 73, 977 (2001)

15. M. Chaichian, M.M. Sheikh-Jabbari, A. Tureanu, Phys. Rev. Lett. 86, 2716 (2001)

16. M. Chaichian, A. Demichev, P. Presnajder, M.M. Sheikh-Jabbari, A. Tureanu, Phys. Lett. B 527, 149 (2002)

17. R.J. Szabo, Phys. Rep. 378, 207 (2003)

18. S. Sivasubramanian, Y.N. Srivastava, G. Vitiello, A. Widom, Phys. Lett. A 311, 97 (2003)

19. M. Chaichian, M.M. Sheikh-Jabbari, A. Tureanu, Eur. Phys. J. C 36, 251 (2004)

20. A. Kokado, T. Okamura, T. Saito, Phys. Rev. D 69, 125007 (2004)

21. O. Bertolami, J.G. Rosa, C.M.L. de Aragăo, P. Castorina, D. Zappalà, Phys. Rev. D 72, 025010 (2005)

22. K. Li, J. Wang, C. Chen, Mod. Phys. Lett. A 20, 2165 (2005)

23. O. Bertolami, J.G. Rosa, C.M.L. de Aragăo, P. Castorina, D. Zappalà, Mod. Phys. Lett. A 21, 795 (2006)

24. N. Khosravi, H.R. Sepangi, M.M. Sheikh-Jabbari, Phys. Lett. B 647, 219 (2007)

25. M. Chaichian, A. Tureanu, G. Zet, Phys. Lett. B 660, 573 (2008)

26. C. Bastos, O. Bertolami, N.C. Dias, J.N. Prata, J. Math. Phys. 49, 072101 (2008)

27. M.M. Sheikh-Jabbari, A. Tureanu, Phys. Rev. Lett. 101, 261601 (2008)

28. C. Bastos, O. Bertolami, Phys. Lett. A 372, 5556 (2008)

29. A. Alves, O. Bertolami, Phys. Rev. D 82, 047501 (2010)

30. C. Bastos, O. Bertolami, N. Costa Dias, J.Nuno Prata, Phys. Rev. D 86, 105030 (2012)

31. C. Bastos, O. Bertolami, N. Dias, J. Prata, Int. J. Mod. Phys. A 28, 1350064 (2013)

32. A.E. Bernardini, O. Bertolami, Phys. Rev. A 88, 012101 (2013)

33. S.-D. Liang, H. Li, G.-Y. Huang, Phys. Rev. A 90, 010102 (2014)

34. O. Bertolami, P. Leal, Phys. Lett. B 750, 6 (2015) 
35. R. Bufalo, A. Tureanu, Phys. Rev. D 92, 065017 (2015)

36. C. Bastos, A.E. Bernardini, O. Bertolami, N. Costa Dias, J.Nuno Prata, Phys. Rev. D 93, 104055 (2016)

37. A.A. Deriglazov, A.M. Pupasov-Maksimov, Phys. Lett. B 761, 207 (2016)

38. A.A. Deriglazov, W. Guzman Ramirez, Adv. Math. Phys. 2017, 7397159 (2017)

39. Kh.P. Gnatenko, V.M. Tkachuk, Phys. Lett. A 381, 2463 (2017)

40. Kh.P. Gnatenko, Europhys. Lett. 123, 50002 (2018)

41. Kh.P. Gnatenko, M.I. Samar, V.M. Tkachuk, Phys. Rev. A 99, 012114 (2019)

42. Kh.P. Gnatenko, Phys. Rev. D 99, 026009 (2019)

43. S. Bellucci, A. Nersessian, C. Sochichiu, Phys. Lett. B 522, 345 (2001)

44. H. Falomir, J. Gamboa, J. Lopez-Sarrion, F. Mendez, P.A.G. Pisani, Phys. Lett. B 680, 384 (2009)

45. M. Gomes, V.G. Kupriyanov, A.J. da Silva, Phys. Rev. D 81, 085024 (2010)

46. A. Das, H. Falomir, J. Gamboa, F. Mendez, M. Nieto, Phys. Rev. D 84, 045002 (2011)

47. H. Falomir, J. Gamboa, M. Loewe, F. Mendez, J.C. Rojas, Phys. Rev. D 85, 025009 (2012)

48. A.F. Ferrari, M. Gomes, V.G. Kupriyanov, C.A. Stechhahn, Phys. Lett. B 718, 1475 (2013)

49. M. Gomes, V.G. Kupriyanov, Phys. Rev. D 79, 125011 (2009)

50. M. Gomes, V.G. Kupriyanov, A.J. da Silva, J. Phys. A Math. Theor. 43, 285301 (2010)

51. A. Fring, L. Gouba, F.G. Scholtz, J. Phys. A 43, 345401 (2010)

52. V.G. Kupriyanov, J. Phys. A Math. Theor. 46, 245303 (2013)

53. V.G. Kupriyanov, J. Math. Phys. 54, 112105 (2013)

54. V.G. Kupriyanov, Phys. Lett. B 732, 385 (2014)

55. V.G. Kupriyanov, Fortschr. Phys. 69, 881 (2014)

56. S.A. Alavi, S. Abbaspour, J. Phys. A Math. Theor. 47, 045303 (2014)

57. S.A. Alavi, N.R. Pramana, J. Phys. 88, 77 (2017)

58. S.A. Alavi, M.Amiri Nasab, Gen. Relativ. Gravit. 49, 5 (2017)

59. S. Dey, A. Fring, Phys. Rev. D 90, 084005 (2014)

60. LTs Adzhemyan, T.L. Kim, M.V. Kompaniets, V.K. Sazonov, Nanosyst. Phys. Chem. Math. 6, 461 (2015)

61. W. Pauli, Z. Phys. 43, 601 (1927)

62. J. Formánek, R.J. Lombard, J. Mares, Czech. J. Phys. 54, 289 (2004)

63. R.J. Lombard, J. Mares, C. Volpe, J. Phys. G Nucl. Part. Phys. 34, 1879 (2007)

64. M. De Sanctis, P. Quintero, Eur. Phys. J. A 39, 145 (2009)

65. R. Yekken, R.J. Lombard, J. Phys. A Math. Theor. 43, 125301 (2010)

66. R. Yekken, M. Lassaut, R.J. Lombard, Ann. Phys. 338, 195 (2013)
67. A. Schulze-Halberg, O. Yesiltas, J. Math. Phys. 59, 113503 (2018)

68. M. Abramowitz, I.A. Stegun, Handbook of Mathematical Functions: with Formulas, Graphs, and Mathematical Tables (Dover Publications, INC., New York, 1965)

69. A. Elbert, J. Comput. Appl. Math. 133, 65 (2001)

70. S.H. Patil, K.D. Sen, Phys. Lett. A 362, 109 (2007)

71. K.B. Oldham, J. Spanier, The Fractional Calculus: Theory and Applications of Differentiation and Integration to Arbitrary Order (Academic Press Inc, Dover Book Publications, New York, 2006)

72. M.D. Ortigueira, Fractional Calculus for Scientists and Engineers (Lecture Notes in Electrical Engineering) (Springer, Dordrecht, 2011)

73. R. Herrmann, Fractional Calculus: An Introduction for Physicists (World Scientific Publishing Company, Singapore, 2014)

74. N. Laskin, Phys. Rev. E 66, 056108 (2002)

75. M. Naber, J. Math. Phys. 45, 3339 (2004)

76. J. Dong, M. Xu, J. Math. Phys. 48, 072105 (2007)

77. M. Jeng, S.-L.-Y. Xu, E. Hawkins, J.M. Schwarz, J. Math. Phys, 51, 062102 (2010)

78. A.N. Hatzinikitas, J. Math. Phys. 51, 123523 (2010)

79. M. Cheng, J. Math. Phys. 53, 043507 (2012)

80. S.S. Bayin, J. Math. Phys. 54, 012103 (2013)

81. B.A. Stickler, Phys. Rev. E 88, 012120 (2013)

82. T. Sandev, I.Petreska Trifce, E.K. Lenzi, J. Math. Phys. 55, 092105 (2014)

83. Z. Xiao, W. Chaozhen, L. Yingming, L. Maokang, Ann. Phys. 350, 124 (2014)

84. Y. Zhang, X. Liu, M.R. Belić, W. Zhong, Y. Zhang, M. Xiao, Phys. Rev. Lett. 115, 180403 (2015)

85. S.S. Bayin, J. Math. Phys. 57, 123501 (2016)

86. D. Zhang, Y. Zhang, Z. Zhang, N. Ahmed, Y. Zhang, F. Li, M.R. Belic, M. Xiao, Annalen der Physik 529, 1700149 (2017)

87. S. Bhattarai, J. Differ. Equ. 263, 3197 (2017)

88. A. Majlesi, H. Roohani Ghehsareh, A. Zaghian, Eur. Phys. J. Plus 132, 516 (2017)

89. J. Li, J. Math. Phys. 58, 102701 (2017)

90. UAl Khawaja, M. Al-Refai, G. Shchedrin, L.D. Carr, J. Phys. A Math. Theor. 51, 235201 (2018)

91. L. Shena, X. Yao, J. Math. Phys. 59, 081501 (2018)

92. M. Chen, S. Zeng, D. Lu, W. Hu, Q. Guo, Phys. Rev. E 98, 022211 (2018)

93. X. Zhang, B. Yang, C. Wei, M. Luo, Commun. Nonlinear Sci. Numer. Simul. 67, 290 (2019)

94. N. Laskin, Fractional Quantum Mechanics (World Scientific, Singapore, 2018)

95. N. Laskin, Phys. Lett. A 268, 298 (2000)

96. N. Laskin, Phys. Rev. E 62, 3135 (2000) 